\title{
Enterococcus faecium TIR-Domain Genes Are Part of a Gene Cluster Which Promotes Bacterial Survival in Blood
}

\author{
Theresa M. Wagner (D), Jessin Janice ${ }^{1},{ }^{1,2}$ Fernanda L. Paganelli, ${ }^{3}$ Rob J. Willems, ${ }^{3}$ \\ Fatemeh Askarian, ${ }^{1}$ Torunn Pedersen, ${ }^{2}$ Janetta Top, ${ }^{3}$ Carla de Haas, ${ }^{3}$ Jos A. van Strijp, ${ }^{3}$ \\ Mona Johannessen $\mathbb{D}^{1}{ }^{1}$ and Kristin Hegstad $\mathbb{D}^{1,2}$ \\ ${ }^{1}$ Research Group for Host-Microbe Interactions, Department of Medical Biology, Faculty of Health Sciences, \\ UiT-The Arctic University of Norway, Tromsø, Norway \\ ${ }^{2}$ Norwegian National Advisory Unit on Detection of Antimicrobial Resistance, \\ Department of Microbiology and Infection Control, University Hospital of North-Norway, Tromsø, Norway \\ ${ }^{3}$ Department of Medical Microbiology, University Medical Center Utrecht, Utrecht University, Utrecht, Netherlands
}

Correspondence should be addressed to Kristin Hegstad; kristin.hegstad@uit.no

Received 12 June 2018; Accepted 14 October 2018; Published 3 December 2018

Academic Editor: Todd R. Callaway

Copyright (c) 2018 Theresa M. Wagner et al. This is an open access article distributed under the Creative Commons Attribution License, which permits unrestricted use, distribution, and reproduction in any medium, provided the original work is properly cited.

\begin{abstract}
Enterococcus faecium has undergone a transition to a multidrug-resistant nosocomial pathogen. The population structure of $E$. faecium is characterized by a sharp distinction of clades, where the hospital-adapted lineage is primarily responsible for bacteremia. So far, factors that were identified in hospital-adapted strains and that promoted pathogenesis of nosocomial E. faecium mainly play a role in adherence and biofilm production, while less is known about factors contributing to survival in blood. This study identified a gene cluster, which includes genes encoding bacterial Toll/interleukin-1 receptor- (TIR-) domain-containing proteins (TirEs). The cluster was found to be unique to nosocomial strains and to be located on a putative mobile genetic element of phage origin. The three genes within the cluster appeared to be expressed as an operon. Expression was detected in bacterial culture media and in the presence of human blood. TirEs are released into the bacterial supernatant, and TirE2 is associated with membrane vesicles. Furthermore, the tirE-gene cluster promotes bacterial proliferation in human blood, indicating that TirE may contribute to the pathogenesis of bacteremia.
\end{abstract}

\section{Introduction}

In the last three decades, the Gram-positive bacterium Enterococcus has undergone a pronounced transition from a human gut commensal to a multidrug-resistant nosocomial opportunistic pathogen [1-3]. Enterococcal infections are mainly caused by Enterococcus faecium and E. faecalis $[4,5]$. E. faecalis historically accounted for $80-90 \%$ of the clinical isolates, and $5-10 \%$ used to be E. faecium [6]. However, E. faecium has risen to be the cause of $34 \%$ of the enterococcal infections [7].

The clade structure of E. faecium displays distinct groups, where commensal strains fall into clade B and hospital-associated strains into clade A $[8,9]$. Enterococcal genomes are highly plastic and are able to acquire and exchange large fragments of DNA. Especially in clade A, the cumulative adaptive gene uptake is reflected by a larger genome size [8].

The most severe infection caused by E. faecium is endocarditis, and additionally, E. faecium often causes urinary tract infection and bacteremia [4]. Infections are established upon translocation to the bloodstream, through the perturbed intestine or uptake from the contaminated hospital environment or skin [4].

The host innate immune system provides the first line of defense against microbial attacks. It is induced through the recognition of highly conserved microbial structures, namely, pathogen-associated molecular patterns (PAMPs), 
by pattern recognition receptors, among which Toll-like receptors (TLRs) are predominant $[10,11]$. The recognition of PAMPs by TLRs mediates antimicrobial responses, such as phagocytosis and microbial killing. Out of ten presently described human TLRs, TLR2 has been identified to be central in recognition of E. faecium [12]. MyD88 is the key intracellular adaptor for TLR2, and stimulation of its intracellular pathway leads to NF-kB activation, resulting in proinflammatory mediator expression [13-15]. Since the initial recognition of a pathogen by immune cells is crucial for all subsequent antimicrobial responses of the host, interference with the innate immune response is a significant advantage for invasive bacteria, and counteraction is through molecular mimicry and immune evasion factors $[11,16]$.

Bacterial TIR- (Toll/interleukin-1 receptor-) domaincontaining proteins structurally mimic host domains, which are crucial for protein-protein interaction in TLR signaling cascade adaptors $[17,18]$. Immune evasive properties of bacterial TIR proteins were first described in Salmonella enterica, where the protein was named "TlpA" (TIR-like protein A) [18]. Since then, TIR proteins with immune evasive properties have been described in a range of Gram-negative and Gram-positive bacteria, including Staphylococcus aureus $[19,20]$ and E. faecalis [21]. In general, bacterial TIR proteins negatively interfere with TLR signaling through adaptor blockade or adaptor degradation [22]. TIR-domain-containing proteins share several amino acid sequence motifs, such as boxes 1 and 2 as well as the WxxxE motif $[22,23]$. The TIR-domain-containing protein in E. faecalis (TcpF) has been described to be predominant among urinary tract infection isolates, attenuate MyD88mediated signaling, and promote bacterial survival within macrophages $[21,24]$. In the closely related E. faecium, however, TIR-domain-containing proteins have not been described yet. The virulence factors so far identified in E. faecium are mainly associated with adhesion, aggregation, growth, and biofilm formation [25-42]. Identification of virulence factors might reveal targets for novel therapeutic approaches.

Our aims were to identify TIR-domain-containing proteins in E. faecium (TirEs), compare their prevalence in nosocomial versus community-associated strains, explore their genetic context, and study their function.

\section{Materials and Methods}

2.1. Bacterial Strains, Plasmids, and Growth Conditions. Prevalence screening for tirE genes and the gene $h p 1$ between the two tirE genes was performed in a diverse E. faecium collection. The strains were of different geographical origin (24 countries), the main countries being the Netherlands (587 isolates), Latvia (96 isolates), Norway (88 isolates), Switzerland (82 isolates), Greece (79 isolates), Germany (61 isolates), Portugal (56 isolates), and Denmark (40 isolates). The total of 1194 strains consist of blood culture (161 isolates), other hospital-associated isolates (meaning feces, urine, or wound, 875 isolates), and human community (158 isolates) isolates (Table S1).
E. faecium and Escherichia coli strains as well as plasmids used for laboratory experiments in this study are listed in Table S2. E. faecium was grown in the brain heart infusion (BHI) medium or Luria-Bertani (LB) medium at $30^{\circ} \mathrm{C}$ or $37^{\circ} \mathrm{C}$ with shaking; E. coli strains were grown in the LB medium or LB supplemented with $20 \mathrm{mM}$ glucose at $37^{\circ} \mathrm{C}$ with shaking.

For E. faecium, the antibiotics gentamicin and spectinomycin were used at a concentration of $300 \mu \mathrm{g} / \mathrm{ml}$ and erythromycin at $25 \mu \mathrm{g} / \mathrm{ml}$. For E. coli, gentamicin and spectinomycin were used at a concentration of $30 \mu \mathrm{g} / \mathrm{ml}$ and $100 \mu \mathrm{g} / \mathrm{ml}$, respectively.

For the Rosetta-gami (DE3) pLysS E. coli protein expression strain, the following antibiotic concentrations were used: ampicillin $100 \mu \mathrm{g} / \mathrm{ml}$, chloramphenicol $50 \mu \mathrm{g} / \mathrm{ml}$, tetracycline $12.5 \mu \mathrm{g} / \mathrm{ml}$, kanamycin $15 \mu \mathrm{g} / \mathrm{ml}$, and streptomycin $50 \mu \mathrm{g} / \mathrm{ml}$. All antibiotics were obtained from Sigma-Aldrich (USA).

\subsection{Bioinformatic Analysis of TIR-Domain-Containing} Proteins. Putative genes encoding TIR-domain-containing proteins were identified in the E. faecium E1162 genome (GenBank: ABQJ00000000), and protein sequences were found in contig107 (ABQJ01000097.1) with locus tags EFME1162_RS19585 (old locus tag EfmE1162_2149) and EFME1162_RS19595 (old locus tag EfmE1162_2151), respectively.

TIR domains and protein families were verified through searches in the Conserved Domain Database (CDD) [43]. Alignment to previously described bacterial and eukaryotic TIR proteins (BtpA from Brucella melitensis [EXU84762.1], TcpC from E. coli [NP_754290], YpTdp from Yersinia pestis [WP_002213208.1], PdTir from Paracoccus denitrificans [WP_011746463.1], TirS from S. aureus [SAS0038, WP_000114516.1], SaTlp1 from S. aureus [CAQ50581.1], TcpF from E. faecalis [CCO72761.1], Myd88 from Homo sapiens [AAH13589.1], and TLR2 from Homo sapiens [AAC34133.1]) was performed in the multiple sequence alignment program MAFFT version 7 [44], and the alignment was viewed and manually curated in AliView [45]. The alignment was illustrated using ESPript 3.x with secondary structure elements of BtpA [46], accessed under 4lzp in the RCSB Protein Data Bank $[47,48]$. A phylogenetic tree was constructed with the algorithm PROTGAMMAAUTO with 100 bootstrap replicates for these protein sequences using RAxML v8.2 [49]. The secondary structures were predicted in the protein fold recognition server Phyre2 [50], superposed to the crystal structure of the TIR domain of human MyD88 using PDBe Fold v2.59 [51], and illustrated in PyMOL [52].

2.3. Detection of Genes by PCR. PCR was performed with gene-specific primers (prevalence screening primers (Table S3)) and DreamTaq Green PCR Master Mix (Thermo Fischer Scientific, USA) according to the manufacturer's instruction. One microliter of bacterial culture in $\mathrm{BHI}$ broth was used as the template. A standard program of $10 \mathrm{~min}$ initial denaturation at $95^{\circ} \mathrm{C}$ followed by 30 cycles of $30 \mathrm{~s}$ 
denaturation at $95^{\circ} \mathrm{C}, 30 \mathrm{~s}$ annealing at $55^{\circ} \mathrm{C}$, and $30 \mathrm{~s}$ elongation at $72^{\circ} \mathrm{C}$ with a final elongation step for $7 \mathrm{~min}$ at $72^{\circ} \mathrm{C}$ was used. PCR products were thereafter visualized on a $1 \%$ agarose gel.

2.4. Phylogenomics. To study the distribution of tirE genes, E. faecium genome assemblies were downloaded on June 27, 2017 ( $n=516)$, from the National Center for Biotechnology Information (NCBI). BLASTp $[53,54]$ searches were performed against all the downloaded genomes for the proteins TirE1 (EFF33949.1), Hp1 (EFF33950.1), and TirE2 (EFF33951.1). A core genome phylogenetic tree was constructed with all E. faecium genomes using Parsnp [55]. The resulting tree was visualized and annotated for the presence or absence of tirE1, $h p 1$, and tirE2 genes, in FigTree v1.4.3 [56]. Metadata including the country of origin, isolation source, disease information, assembly level, and antimicrobial-resistant phenotypes were collected for each of the tirE-positive genomes (Table S4) from NCBI and PATRIC [57].

2.5. Identification of a Putative Mobile Genetic Element. The genetic regions up- and downstream of the tirE locus in the E. faecium E1162 genome sequence were compared to the corresponding regions of the community strain $E$. faecium 17OM39, which lacks the tirE region. The comparison was viewed and analyzed using Artemis Comparison Tool [58]. Pairwise comparison figures were drawn using EasyFig 2.2.2 $[59,60]$. Insertion of a putative mobile genetic element in E1162 was identified, and each gene in this region was subjected to BLAST [53] and CDD [43] searches to identify the similar proteins and protein domains and to Phyre2 [50] for structural, functional, and evolutionary classification prediction (Table 1).

The inserted genetic region that is flanked by phage integrases was extracted from all the tirE-locus-positive genomes. Genomes, which contained incomplete insertion elements, because of assembly incompleteness, were excluded from further analyses. The mobile genetic elements were reannotated in Prokka 1.11 [61], and proteins were clustered with Roary 3.8.2 [62]. To understand the conserved nature of these elements, a heat map was created and visualized in GI tools [63, 64].

2.6. $m R N A$ Expression Analysis by Reverse Transcription (RT) $P C R$. For blood exposure, bacteria (E1162 and K60-39) were grown in $\mathrm{BHI}$ to $\mathrm{OD}_{600} 0.4$, washed with PBS, and resuspended in the Roswell Park Memorial Institute (RPMI) medium with $0.05 \%$ human serum albumin (RPMI-HSA). Fresh human blood was collected from healthy volunteers in hirudin blood tubes (Roche Diagnostics, Switzerland), added to the bacteria to a final concentration of $80 \%$, and incubated on a turning wheel at $37^{\circ} \mathrm{C}$ for $3 \mathrm{~h}$. Blood cells were lysed in $0.3 \%$ ice-cold saponin, and the bacterial pellet was washed with PBS. All bacterial samples were stored in RNAprotect solution (Qiagen, Germany). RNA was extracted using the RNeasy Mini Kit (Qiagen, Germany) following the manufacturer's instructions with a prolonged initial lysis step using mutanolysin $(0.1 \mathrm{U} / \mu \mathrm{l})$ and lysozyme $(1 \mathrm{mg} / \mathrm{ml})$ for $1 \mathrm{~h}$ at $37^{\circ} \mathrm{C}$. After DNase treatment (Heat and Run Kit; ArcticZymes, Norway), RNA integrity and quantity were checked by NanoDrop as well as on agarose gel. Reverse transcription was performed (High-Capacity cDNA Reverse Transcription Kit; Applied Biosystems, USA) on 100 ng RNA, and DNA contamination was ruled out through minus reverse transcriptase $(-\mathrm{RT})$ control. The genes were thereafter amplified from the cDNA through PCR (prevalence screening primers (Table S3); 30 cycles; annealing temperature $55^{\circ} \mathrm{C}$ ), and the products were visualized on a $1 \%$ agarose gel.

In order to assess whether the genes are expressed together, junction primers were used on the cDNA template to amplify the intergenic regions (junction primers (Table S3); 30 cycles; annealing temperature $55^{\circ} \mathrm{C}$ ). Additionally, the internal primers (prevalence screening primers (Table S3); 30 cycles; annealing temperature $55^{\circ} \mathrm{C}$ ) were combined to link the gene product. The identity of gene products was confirmed by sequencing the PCR products.

2.7. Cloning, Expression, and Purification of Recombinant N-His-TirE. The E1162 tirE1, hp1, and tirE2 genes were amplified with BamHI-Fw and NotI-Rv primers (expression primers (Table S3)) and cloned in frame into the expression plasmid pRSETB containing an N-terminal $\mathrm{HIS}_{6}$ tag (Invitrogen, USA) (Table S3), as previously described [65]. After verification of the correct sequence by DNA sequencing, the pRSET/TirE1, pRSET/Hp1, and pRSET/TirE2 constructs were transformed into E. coli Rosetta-gami (DE3) plysS. Expression was induced in the mid-logarithmic growth phase with $1 \mathrm{mM}$ isopropyl $\beta$-D-1thiogalactopyranoside (IPTG) for $4 \mathrm{~h}$ or overnight (Figure S1A).

Proteins were isolated from an HiTrap Chelating HP column under either native (Figure S1B) or denaturing (8 M urea (Figure S1C)) conditions and eluted using an imidazole gradient, ranging from 10 to $500 \mathrm{mM}$ in an ÄKTA FPLC protein purification system (GE Healthcare Life Sciences, Australia). The protein-containing fractions were pooled. Fractions containing denaturated proteins were refolded through dialysis with salt buffer $(50 \mathrm{mM}$ Tris and $30 \mathrm{mM}$ $\mathrm{NaCl}, \mathrm{pH}$ 7.8). Finally, proteins from both native and denaturating conditions were dialyzed with PBS. The purity of the proteins was confirmed by SDS-PAGE. The calculated protein sizes with the his tag were TirE1 $20.4 \mathrm{kDa}$, TirE2 $34.9 \mathrm{kDa}$, and $\mathrm{Hp} 156.9 \mathrm{kDa}$.

\subsection{Anti-TirE Serum, Immunoprecipitation, and Western} Blot. Purified TirE1, Hp1, and TirE2 were then used to immunize Enterococcus-negative rabbits by Eurogentec (Belgium). Preimmune serum was tested for nonreactivity towards enterococcal lysates prior to injection.

Bacteria (E1162 and K60-39) from $25 \mathrm{ml}$ overnight culture were pelleted and resuspended in the lysis buffer (lysozyme $10 \mathrm{mg} / \mathrm{ml}$ and mutanolysin $6 \mathrm{U} / \mu \mathrm{l}$ ), incubated for $1 \mathrm{~h}$ at $37^{\circ} \mathrm{C}$, and sonicated (4 times $1 \mathrm{~min}$ on-off). 
TABle 1: ORFs in the putative MGE-TirE of E. faecium strain E1162.

\begin{tabular}{|c|c|c|c|c|c|c|}
\hline Locus tag in E1162 & Product NCBI & $\begin{array}{l}\text { CDD (Conserved } \\
\text { Domain Database) }\end{array}$ & $\begin{array}{l}\text { Phyre } 2 \text { prediction } \\
\text { (putative function) }\end{array}$ & $\begin{array}{l}\text { SignalP prediction } \\
\text { (secretion signal) }\end{array}$ & Size $(\mathrm{Da})$ & Name \\
\hline EFME1162_RS19580 & Site-specific integrase & DNA-binding integrase & Integrase & - & 44551 & int1 \\
\hline EFME1162_RS19585 & - & TIR-like & TIR domain & - & 18496 & tirE1 \\
\hline EFME1162_RS19590 & - & Sir2-like & Nucleotide binding & - & 55003 & hp1 \\
\hline EFME1162_RS19595 & - & TIR-like & TIR domain & - & 32901 & tirE2 \\
\hline EFME1162_RS19600 & Transcription regulator & DNA binding & DNA binding & - & 9466 & hp2 \\
\hline EFME1162_RS19605 & - & - & - & Yes & 29307 & hp3 \\
\hline EFME1162_RS0109495 & - & - & - & - & 12407 & hp4 \\
\hline EFME1162_RS19610 & - & - & - & - & 8588 & hp5 \\
\hline EFME1162_RS19615 & - & DnaJ super family & Transcription $\mathrm{r}$ & Yes & 98796 & hp6 \\
\hline EFME1162_RS0109500 & - & - & - & - & 8445 & hp7 \\
\hline EFME1162_RS19620 & - & - & DNA bin & - & 34840 & hp8 \\
\hline EFME1162_RS19625 & DNA binding protein & DNA bin & DNA binding & - & 11591 & hp9 \\
\hline EFME1162_RS19630 & Transcription regulator & Transcription regulator & Transcription regulator & - & 31516 & hp10 \\
\hline EFME1162_RS19635 & Site-specific integrase & Integrase & Integrase & - & 44970 & int2 \\
\hline
\end{tabular}

The bacterial supernatant was concentrated 40 times by centrifugation using a $10 \mathrm{kDa}$ cutoff filter and sonicated (4 times $1 \mathrm{~min}$ on-off). Immunoprecipitation was performed using SureBeads Protein G magnetic beads (Bio-Rad, USA) according to the manufacturer. In brief, the beads were washed with PBS-T (PBS with $0.01 \%$ Tween), and 50\% antiTirE1, anti-TirE2, or anti-Hp1 serum in PBS was added. After washing three times, the bacterial lysate or supernatant was incubated with the beads for $1 \mathrm{~h}$ at room temperature under shaking. Finally, the samples were eluted into $15 \mu \mathrm{l}$ western blot sample buffer.

Western blot was performed as described previously [66]. For immunodetection, the polyclonal antiserum against TirE1 (1:300), Hp1 (1:300), and TirE2 (1:300) was used. Swine horseradish peroxidase- (HRP-) conjugated anti-rabbit IgG antibodies $(1: 3000)$ were used as secondary antibodies.

2.9. Membrane Vesicle Isolation from Enterococcus. Membrane vesicles were isolated from E. faecium as described earlier [67]. In short, bacteria (K60-39 and DO) were grown to the stationary phase in $\mathrm{LB}$, and bacterial cells were removed by spinning down the culture for $30 \mathrm{~min}$ at $4^{\circ} \mathrm{C}$ at $6000 \mathrm{~g}$. The supernatant was filtrated through $0.22 \mu \mathrm{m}$ vacuum-bottle top filters (Millipore, USA). A vesicular pellet was then obtained from the supernatant through ultracentrifugation at $4^{\circ} \mathrm{C}$ at $100.000 \mathrm{~g}$ for $4 \mathrm{~h}$, washed with PBS, and centrifuged again at $4^{\circ} \mathrm{C}$ at $100.000 \mathrm{~g}$ for $4 \mathrm{~h}$. The vesicular pellet was thereafter cleaned through density gradient centrifugation using OptiPrep (Sigma-Aldrich, USA), where the MVcontaining samples were bottom loaded. $200 \mu \mathrm{l}$ fractions were analyzed in SDS-PAGE, and vesicle-containing fractions were selected. Proteins were TCA precipitated from the respective fractions and analyzed in a Thermo Scientific Q-Exactive mass spectrometer (Thermo Fisher Scientific, USA) after solution trypsinization. The raw data were processed in Proteome Discoverer 2.1 software. The fragmentation spectra were searched against WGS data of the strain itself (E. faecium DO and K60-39) using the Sequest HT program.
2.10. Markerless Mutant Construction. Utilizing the Cre-lox recombination system, markerless gene deletion mutants in the tirE gene cluster (locus_tag: EFME1162_RS19585 to EFME1162_RS19595) were created as previously described [40, 68, 69]. In short, gblock (TirE1_up-Tir2_down (Table S3)) consisting of the $5^{\prime}$ and $3^{\prime}$ flanking regions (approximately 500 bp each) of the target with an EcoRI site between both fragments was amplified with tirE1_up and tirE2_down primers (Table S3) and cloned into the vector pWS3 [70], resulting in pDELtir. Then, a gentamicin-resistant cassette flanked by lox66 and lox71 sites [69] was cloned into the EcoRI site included in the TirE1_up-Tir2_down gblock between the $5^{\prime}$ and $3^{\prime}$ flanking regions in pDELtir.

The resulting plasmid pDELtirGenta was subsequently electroporated in competent E. faecium E1162, as previously described. Marked mutants were obtained by growing the gentamicin-resistant transformants at appropriate temperatures supplemented with respective antibiotics. The plasmid pWS3-Cre carrying a gene encoding the Cre recombinase was introduced into the marked mutant by electroporation. Subsequent culturing to remove the gentamicin-resistant cassette and thereby ensure loss of pWS-Cre was performed as previously described [69]. Excision of the gentamicin-resistant cassette and loss of pWS3Cre were verified by PCR using the primers tirE1-check-up and tirE1-check-down, in RT-qPCR using internal primers (prevalence screening primers (Table S3)) as well as through whole-genome sequencing (WGS) which also ensured that no other mutations had occurred.

Genomic DNA of the markerless mutant was isolated using the Wizard Genomic DNA Purification Kit (Promega, USA) with lysozyme $(20 \mathrm{mg} / \mathrm{ml})$, dissolved in water, and sequenced by the HiSeq 2500 platform (Illumina, USA).

E. faecium and its knockout mutant genomes were compared pairwise using Artemis Comparison Tool [58]. Pairwise comparison figures were drawn using EasyFig 2.2.2 [59]. GATK was used for variant calling to estimate the SNP differences between these two variants $[71,72]$.

2.11. GFP-Expression Plasmid in Enterococcus. A pEF25 vector with GFP cloned in the SmaI site resulting in 
pEF451 was transformed into E. coli EC1000, as described earlier (under submission [73]) and electroporated into competent E. faecium E1162 and the markerless isogenic mutant E1162 $\Delta$ tirE. Transformants (GFP-E1162 and GFP-E1162 $\Delta$ tirE) were selected under antibiotic pressure and confirmed by PCR as well as by a confocal laser scanning microscope (CLSM Leica SP5), equipped with an oil plan-Neofluar ( $\times 63 / 1.4$ objective). GFP was excited at $395 \mathrm{~nm}$.

2.12. Cell Lines and Culture. Peripheral blood mononuclear cells (PBMCs) $\left(1 \times 10^{7}\right.$ cells $\left./ \mathrm{ml}\right)$ and human neutrophils $(\mathrm{PMN})\left(5 \times 10^{6}\right.$ cells $\left./ \mathrm{ml}\right)$ were isolated from heparinized blood as described previously [65].

All mammalian cells were grown at $37^{\circ} \mathrm{C}$ in an incubator with 5\% $\mathrm{CO}_{2}$. HEK293T cells, a human embryonic kidney cell line obtained from ATCC, USA, were used to stably express human TLR2, as described previously (under submission [74]). In short, human TLR2 (NM_003264.3) was cloned and its signal peptide (aa1-20) was replaced by the PreProTrypsin signal peptide (MSALLILALVGAAVA), adapted from the pFLAGCMV-1 vector (Sigma-Aldrich, USA). To the N-terminus, a FLAG tag (DYKDDDDK) followed by a flexible linker (GGS) was attached. For stable expression in HEK293T cells, a lentiviral expression system was used. The FLAG tag was detected using anti-FLAG M2 antibody (Sigma-Aldrich, USA), subsequently stained with phycoerythrin-labeled goat-anti-mouse antibody, and detected on a flow cytometer (FACSVerse, BD Biosciences, USA). The HEK293T-TLR2 cells were cultured in Dulbecco's modified Eagle's medium (DMEM) with 10\% (v/v) fetal calf serum (FCS) (Invitrogen Life Technologies, USA) and 100 units/ml of penicillin and streptomycin (SigmaAldrich, Germany).

Thp-1 cells (ATCC, USA), a human monocytic cell line, were maintained in the RPMI 1640 medium with $2 \mathrm{mM}$ L-glutamine supplemented with $10 \%(\mathrm{v} / \mathrm{v})$ fetal bovine serum (FBS; Biowest, USA), $10 \mathrm{mM}$ HEPES, $1 \mathrm{mM}$ sodium pyruvate, $4.5 \mathrm{~g} / \mathrm{L}$ glucose, and $0.05 \mathrm{mM}$ betamercaptoethanol (all from Gibco, Life Technologies, USA). For differentiation of Thp-1 to macrophage-like cells, RPMI 1640 with $2 \mathrm{mM}$ L-glutamine without phenol red was used with the same supplements as above, but with addition of $25 \mathrm{nM}$ phorbol 12-myristate 13-acetate (PMA).

2.13. Cytokine Production upon E. faecium Infection. The effect of the tirE locus on cytokine release was investigated by measuring IL- 8 release upon Thp- 1 infection with the E. faecium wildtype strain E1162 or E1162 $\Delta$ tirE.

Bacteria were grown in $\mathrm{BHI}$ to $\mathrm{OD}_{600} 0.4$, washed with $\mathrm{PBS}$, and resuspended in the cell culture medium and diluted to the appropriate $\mathrm{CFU} / \mathrm{ml}$.

Thp-1 cells were seeded in a 24 -well plate in RPMI at $1 \times$ $10^{5}$ cells per well and differentiated for $24 \mathrm{~h}$ by addition of $25 \mathrm{nM}$ PMA. For infection, $100 \mu \mathrm{l}$ of bacterial suspension (equal to MOI 300, MOI 100, and MOI 10) was added per cell culture well and incubated for $37^{\circ} \mathrm{C}$. The supernatant was collected after 2,4 , and $6 \mathrm{~h}$, and debris was spun down at $13000 \mathrm{rpm}$ at $4^{\circ} \mathrm{C}$.

IL-8 within the supernatant was detected using the IL-8 ELISA Kit (Thermo Fisher Scientific, USA) following the manufacturer's instructions. Absorbance was measured at $450 \mathrm{~nm}$.

2.14. Interference with Ligand-Induced Cytokine Production. Mammalian cells were plated out at $2.5 \times 10^{4}$ cells/well in a 96-well culture plate $24 \mathrm{~h}$ prior to the experiment to reach confluence. The inhibitor (TirEs and/or Hp1 or SSL3 [75]) was added at a final concentration of $10 \mu \mathrm{g} / \mathrm{ml}$, and the cells were incubated at $37^{\circ} \mathrm{C}$ for $1 \mathrm{~h}$. After $6 \mathrm{~h}$ incubation with stimulus (3 ng/ml MALP-2 (Santa Cruz, USA); $3 \mathrm{ng} / \mathrm{ml}$ PAM-2-Cys and $3 \mathrm{ng} / \mathrm{ml}$ PAM-3-Cys (EMC Microcollections, Germany), the supernatant was collected and IL-8 was measured using a specific IL-8 ELISA Kit (Sanquin, the Netherlands) following the manufacturer's instructions. In short, Nunc MaxiSorp plates were coated with anti-IL-8, washed and blocked with $4 \%$ nonfat dry milk in PBS-T, and washed again and incubated with the supernatant in high-performance ELISA (HPE) buffer at $37^{\circ} \mathrm{C}$ for $1 \mathrm{~h}$. After washing, biotinylated anti-IL-8 and subsequently streptavidin-HRP were added. Tetramethylbenzidine (TMB) was used as the substrate, and the reaction was stopped with sulfuric acid as soon as a change in color was visible. $\mathrm{OD}_{450}$ was measured for quantification.

2.15. Serum-Dependent Phagocytosis in Flow Cytometry. Blood was collected from healthy volunteers and allowed to clot. After centrifugation, the human serum was collected and pooled. The serum was stored at $-70^{\circ} \mathrm{C}$. When required, heat inactivation of serum was performed at $56^{\circ} \mathrm{C}$ for $30 \mathrm{~min}$.

GFP-E1162 and GFP-E1162 $\Delta$ tirE were grown in BHI to $\mathrm{OD}_{600}$ 0.4, washed with PBS, and exposed to serum for $15 \mathrm{~min}$ at $37^{\circ} \mathrm{C}$ in a 96 -well round bottom plate (Greiner, Austria) on a shaking plateau prior to phagocytosis.

PMNs and PBMCs $(200 \mu \mathrm{l}$ and $300 \mu \mathrm{l}$, respectively) were added to the bacteria, and the cells were coincubated for $15 \mathrm{~min}$ at $37^{\circ} \mathrm{C}$. The cells were thereafter fixed in $1.5 \%$ paraformaldehyde in RPMI-HSA, and fluorescence was measured through flow cytometry (FACSCalibur; Becton Dickinson, USA).

\subsection{Intracellular Survival within Macrophages.} Intracellular survival was assessed in a gentamicinvancomycin protection assay. Thp-1 cells were seeded in a 12-well plate in RPMI with the required supplements at $1 \times$ $10^{6}$ cells per well and differentiated for $24 \mathrm{~h}$ by addition of $25 \mathrm{nM}$ PMA. Bacteria were grown in $\mathrm{BHI}$ to $\mathrm{OD}_{600} 0.4$, washed with PBS, and resuspended in the cell culture medium. For infection, $200 \mu \mathrm{l}$ of bacterial suspension was added per cell culture well and incubated for $1 \mathrm{~h}$. After PBS washing of Thp-1 cells, $16 \mu \mathrm{g} / \mathrm{ml}$ vancomycin and $150 \mu \mathrm{g} / \mathrm{ml}$ 
gentamicin were added to the wells in order to kill the extracellular bacteria. Thp-1 cells were thereafter harvested after $24,48,72$, and $120 \mathrm{~h}$ and lysed in $0.3 \%$ saponin. The released intracellular bacteria were plated on blood agar plates and counted.

2.17. Whole-Blood Phagocytosis and Blood Survival Assay. GFP-E1162 and GFP-E1162 $\Delta$ tirE from a blood agar plate were diluted in $\mathrm{BHI}$ to $\mathrm{OD}_{600} 0.1$ and grown at $37^{\circ} \mathrm{C}$ to $\mathrm{OD}_{600}$ 0.4 , washed with PBS, and resuspended in RPMI-HSA. Blood was obtained from a healthy volunteer in a hirudin blood tube (Roche Diagnostics, Switzerland) and diluted in RPMI-HSA. For all whole-blood experiments, blood was used at $80 \%$ if not otherwise indicated.

Phagocytosis was performed in round-bottom polystyrene tubes under rigorous shaking at $37^{\circ} \mathrm{C}$ for $20 \mathrm{~min}$. Erythrocytes were lysed by adding FACS lysing solution (BD) for $20 \mathrm{~min}$ at $25^{\circ} \mathrm{C}$. The remaining immune cells were washed with RPMI-HSA and fixed in $150 \mu \mathrm{l} 1 \%$ paraformaldehyde in RPMI-HSA. Fluorescence was measured through flow cytometry (FACSCalibur; Becton Dickinson, USA).

For blood survival assays, E1162 and E1162 $\Delta$ tirE were grown in $\mathrm{BHI}$ to $\mathrm{OD}_{600} 0.4$, washed with $\mathrm{PBS}$, and incubated with $80 \%$ fresh hirudin blood in siliconized tubes (SigmaAldrich, USA) on a turning wheel at $37^{\circ} \mathrm{C}$ for $3 \mathrm{~h}$, if not otherwise indicated. Blood cells were lysed in $0.3 \%$ saponin, and CFUs were counted on blood agar plates.

2.18. Statistical Analysis and Data Validation. Statistical data analysis was performed in GraphPad Prism 7. The presented data are expressed as mean \pm SEM of pooled experiments. Blood experiments were performed at least in 3 different donors, representing both male and female.

2.19. Ethics Statement. The human blood analysis was carried out in accordance with ethical principles of the Helsinki Declaration, the Medical Ethics Committee of the University Medical Center Utrecht (the Netherlands), and ethical approval of (2014/1653) REK North-Norway with written informed consent of participants.

\section{Results}

3.1. Identification and Predicted Tertiary Structure of TirEs. Two putative proteins annotated with a TIR domain were identified in E. faecium in the protein database at NCBI. These two open reading frames (ORFs) were found, with a hypothetical protein ( $h p 1)$ in between them. In E. faecium E1162, the TIR-domain-containing locus tags EFME1162_RS19585 and EfmE1162_RS19595 span 492 and $864 \mathrm{bp}$, respectively. In line with previous studies [19], the protein-encoding genes were designated tirE1 and tirE2. The predicted mass of TirE1 was $18.5 \mathrm{kDa}$, TirE2 $32.9 \mathrm{kDa}$, and Hp1 $55.0 \mathrm{kDa}$ (Table 1). The TIR-domains were verified through the CDD search [43] and found to belong to the protein families pfam08937 (TirE1) and pfam13676 (TirE2).
Amino acid sequence alignment of multiple bacterial TIR-domain-containing proteins showed high conservation of the TIR domain across species. Amino acid sequence was particularly conserved in boxes 1 and 2, as well as in the WxxxE motif. In TirE1, the WxxxE motif has a tyrosine instead of tryptophan, but both amino acids are aromatic. The WxxxE motif has been found in most bacterial TIRdomain-containing proteins including PdTir, SaTlp1, and TlpA, as well as eukaryotic TLR proteins TLR1, 2, 4, 6, and 10 and TIR adaptor protein SARM [76] (Figure 1(a)). Variability was observed in the region between strand $\beta \mathrm{B}$ and helix $\alpha \mathrm{B}$, the so-called BB loop (Figure 1(a)). The indicated $\mathrm{BB}$ loop has been described to be surface-exposed and essential for homotypic TIR-domain interactions in TLR/IL-1R signaling [77]. The predicted tertiary structure of both TirEs (Figure 1(b)) shows a typical flavodoxin-like TIR fold, with central parallel $\beta$-sheets surrounded by $\alpha$-helices on both sides of the sheet [77].

A phylogenetic tree illustrating the relatedness of the aligned TIR-domain-containing proteins to each other showed that the human TLR2 and MyD88 are grouped in the same cluster, whereas the bacterial TIR-domain-containing proteins group in different clusters (Figure S2). Even though both TirE1 and TirE2 originate from E. faecium, they fall into two different clusters, indicating that these are probably not just gene duplications but two different genes.

3.2. The tirE Locus Is Prevalent in Nosocomial but Absent in Community Isolates. A total of 1194 E. faecium isolates were screened for tirE1, tirE2, and $h p 1$ either by regular PCR using prevalence screening primers or by BLAST searches in sequenced isolates. tirE1, $h p 1$, and tirE2 genes were found to be highly prevalent in blood culture isolates (tirE1 37\%, hp1 36\%, and tirE2 35\%), and among those, the dominant STs were ST17, ST203, and ST192. The genes were also frequent in other hospital-associated samples (tirE1 32\%, hp1 22\%, and tirE2 31\%) with the most prevalent STs being ST78, ST389, and ST192. 84 of the hospital screening samples were positive for tirE1 and tirE2, but lacked $h p 1$, which coincides with the presence of $v a n B$ vancomycin-resistant cluster in 79 of them (Table S1). Contrasting the high prevalence in blood culture isolates and other hospital-associated samples, the three genes were absent in community isolates (Figure 2).

Furthermore, BLAST search revealed that the tirE locus was found in $14 \%$ of 460 draft genome sequences of nosocomial strains, while these genes were absent in draft genome sequences of all 56 community strains (Figure S3).

This dichotomy in the presence of the tirE locus between hospital and community isolates suggests that the possession of the tirE locus is beneficial for the bacterium in the hospital setting.

3.3. The tirE Locus Is Localized on a Putative Mobile Genetic Element of Phage Origin. Analysis of the genetic surroundings of the tirE locus suggested that the genes were localized on a putative mobile genetic element flanked by two integrases. This putative MGE was designated MGE- 


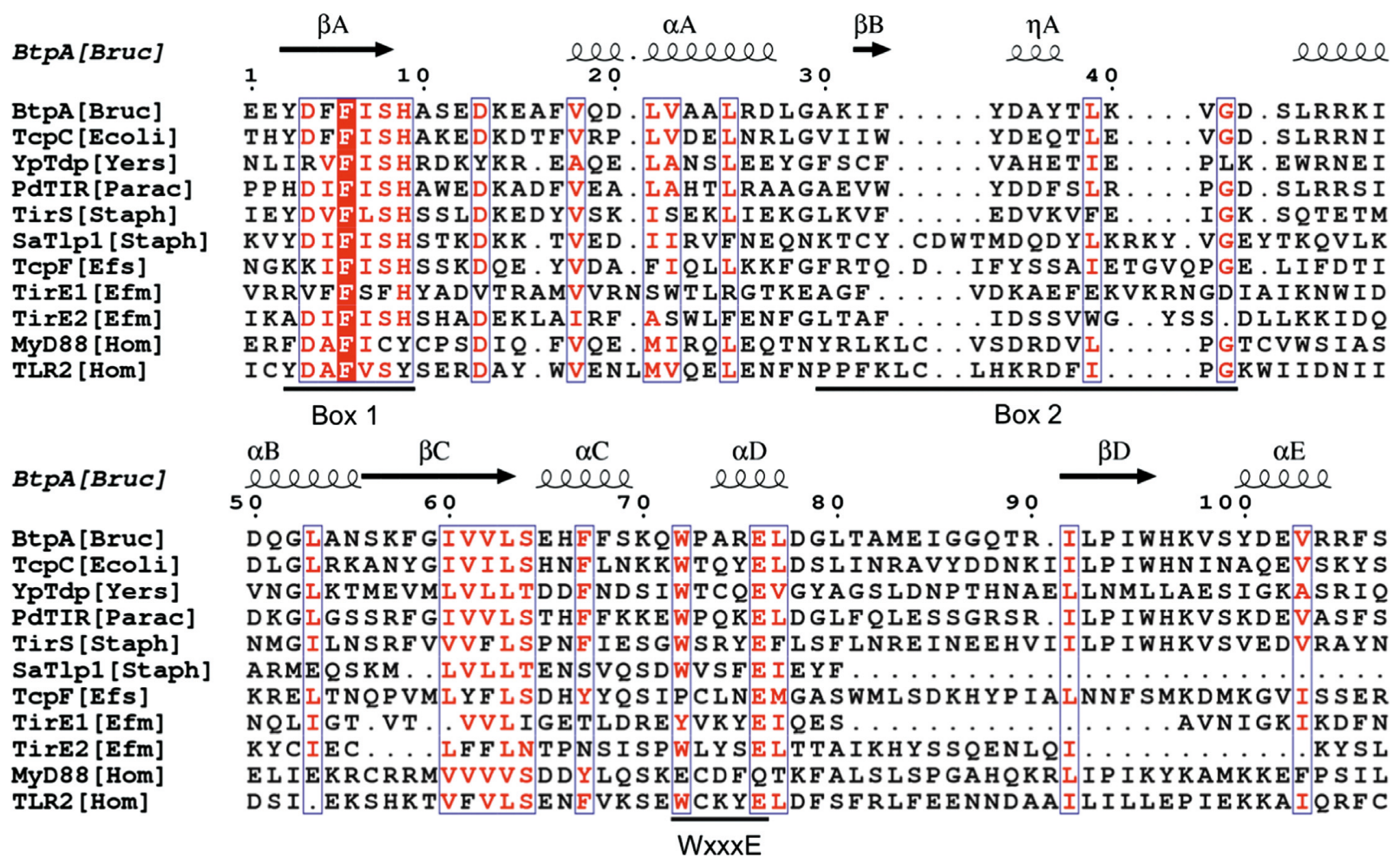

(a)
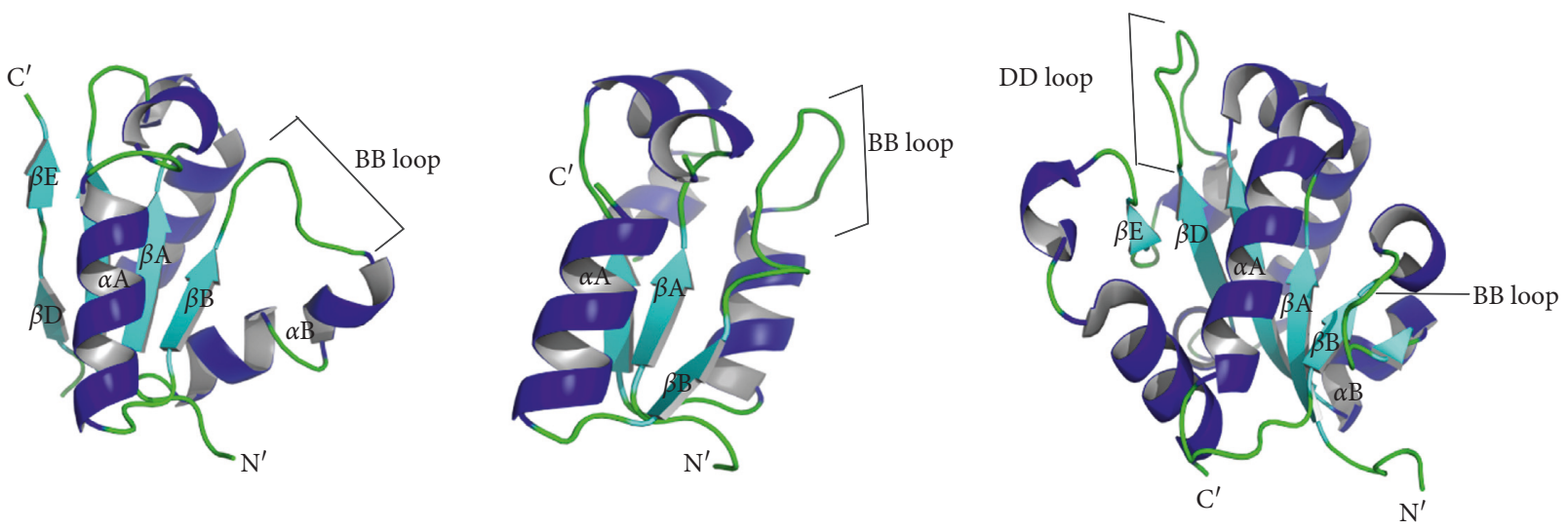

(b)

FIgure 1: Secondary and tertiary structure prediction of TirEs. (a) Primary amino acid sequence alignment of TirE1 and TirE2 to bacterial TIR-domain-containing proteins and human TIR-domain-containing proteins. The region shown displays the TIR domain as predicted by CDD. Similar and identical residues are depicted in red and boxed in blue. Conservation in regions such as Box1, Box2, and the WxxxE motif is marked. The corresponding secondary structure elements of BtpA as determined by X-ray crystallography are shown on top of the alignment. (b) Predicted tertiary structures of TirE1 and TirE2 compared to the crystal structure of the TIR domain of MyD88. Key secondary structures are labeled; $\alpha$-helices are shown in blue, $\beta$-sheets in cyan, and loops in green. The BB loop as a divergent structure is marked.

TirE (for mobile genetic element containing tirEs). Pairwise comparison of the genomic region encompassing MGE-TirE in the strain E1162 with the community strain E. faecium 17 OM39 which does not contain the tirE locus revealed the MGE-TirE's size of $14.425 \mathrm{kbp}$ and site of integration (Figure $3(\mathrm{a}))$. The putative function of the ORFs $(n=13)$ within MGE-TirEs is indicated in Figure 3(a) and in Table 1. Six of the ORFs were predicted to encode either transcription regulators $(n=2)$ or DNA-binding proteins $(n=$
4). $31 \%$ the GC content of MGE-TirE is especially low (GC content calculated in 4 representative strains), since the average GC content for E. faecium is 38\% [78]. The two ORFs flanking MGE-TirE were predicted to encode putative integrases. They both showed high similarity to phage integrases. Furthermore, one of the hypothetical proteins (hp9) was similar to a Lactobacillus plantarum prophage remnant LP4 protein [79]. BLAST analyses of the region as a whole did not show any similarities to the sequences in the 


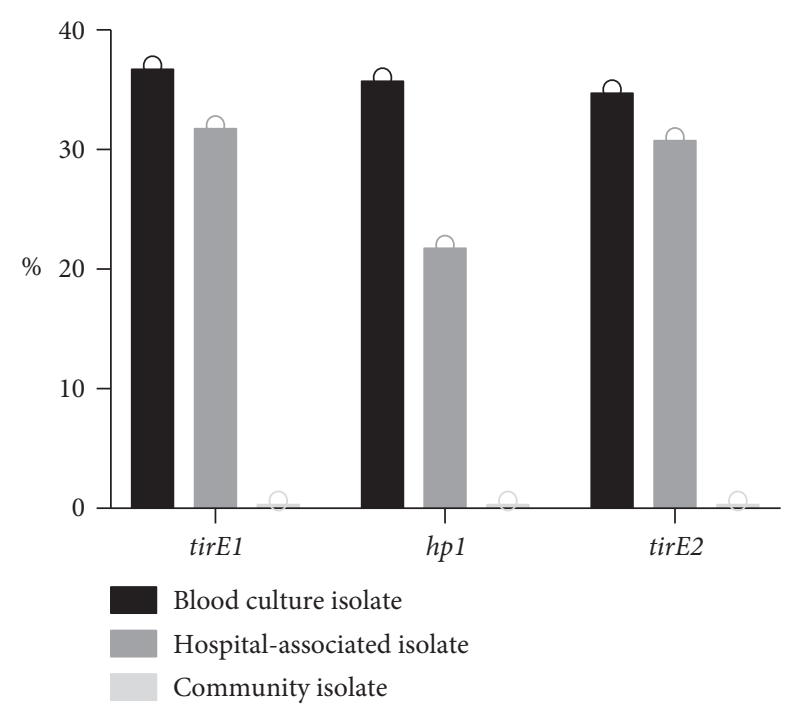

FIgUre 2: Prevalence of the genes tirE1, $h p 1$, and tirE2 in human E. faecium isolates. Percentage indicates the amount of isolates positive for tirE1, $h p 1$, and tirE2 among blood culture isolates $(n=$ $161)$, other hospital-associated isolates $(n=875)$, and community isolates $(n=158)$.

NCBI nonredundant database or the Sequence Read Archive. The deviant GC value and putative function of ORFs indicated presence of foreign genes, possibly of phage origin.

MGE-TirE was probably acquired multiple times during E. faecium evolution, as it occurs in different branches of the core genome phylogenetic tree (Figure S3). Moreover, MGE-TirE was integrated at an identical site adjacent to the host phage integrase gene (int1) in all the TirE-positive genomes, indicating site-specific integration. To describe the conservation of the content of MGE-TirE, a heat map was created, illustrating that most of the genes identified as part of MGE-TirE were highly abundant within the individual elements of all MGE-TirE-containing strains, including the integrases and TIR-domain-containing proteins (Figure 3(b)).

3.4. Expression of Genes of the tirE Locus. RT-PCR revealed that the genes of the tirE locus are expressed in both E1162 and $\mathrm{K} 60-39$. Expression was detected in the stationary phase in $\mathrm{BHI}$ and upon exposure to human blood for $3 \mathrm{~h}$; all three genes showed enhanced expression in E1162 (Figure 4). Promoter prediction analysis revealed only weak promoters between the three genes of the tirE locus, so a linking RTPCR, where the chosen primers hybridize to the different genes, was performed to determine whether the genes are part of a single operon (Figure 5(a)). The junctions of tirE1 and $h p 1$ as well as $h p 1$ and tirE2 gave products (Figure 5(b)), whereas the outward-pointing junctions did not (data not shown). Sequencing confirmed the identity of the PCR products (data not shown). This indicates that the two tirEs and $h p 1$ encoded by the same strand are transcribed together.
3.5. tirE-Locus-Encoded Proteins Are Released into Supernatant and Associated with Membrane Vesicles. Since there were no signal peptide cleavage sites predicted by SignalP in TirEs and Hp1, it was an open question whether bacteria were able to release these proteins (Table 1). Antibodies against TirEs and $\mathrm{Hp} 1$ were specific but unfortunately had low sensitivity (results not shown). In order to detect protein release, anti-TirE/Hp1 serum was used to immunoprecipitate the proteins from the bacterial lysate and supernatant. The immunoprecipitated proteins were thereafter detected by immunoblot. All three proteins were detected in the cell lysate as well as in the supernatant of both E1162 and K60-39 cultures, indicating release through an unknown mechanism (Figures 6(a)-6(c)).

Membrane vesicles (MVs) have previously been suggested as a secretory system [80]. TirE2 and Hp1 were found to be associated with MVs from K60-39 and DO. The exponentially modified protein abundance index (emPAI) value for TirE2 was 0.389 and for HP1 was 0.089 (Figure 6(d)). The identification confidence was characterized as "high" in the protein false discovery rate (FDR) confidence, and the FDR was lower than $1 \%$ with 1 peptide and 2 peptide-to-spectrum matches [67].

3.6. Construction of a tirE1-hp1-tirE2 Knockout Strain for Functional Studies. A markerless mutant lacking the tirE locus was constructed and confirmed by PCR, gene expression, and whole-genome sequencing (Figures S4A-C). Comparison of the whole-genome sequences of the wildtype strain (E1162) and mutant strain (E1162 $\Delta$ tirE) confirmed the absence of tirE1-hp1-tirE2 (Figure S4C), and except for these three genes, the strains share $99 \%$ nucleotide identity (data not shown). The deletion of tirE1-hp1-tirE2 did not affect the bacterial growth in BHI (Figure S4D). Unfortunately, we did not succeed in constructing single gene knockouts nor in complementing the deletion, probably because there were repetitive sequences in this gene region and it has a very low GC content.

3.7. The tirE Locus Does Not Interfere with Cytokine Release. Differentiated Thp1 cells were infected with E1162 or its isogenic mutant E1162 $\Delta$ tirE at MOI 10, 100, and 300. The IL-8 was then detected in the supernatant. As seen in Figure 7(a), E1162 and E1162 $\Delta$ tirE at all MOIs induced IL-8 release in a similar way.

To examine whether TirEs are capable of interfering with TLR2 signaling, HEK293T cells stably expressing TLR2 were stimulated with the synthetic lipopeptides MALP-2, Pam2Cys, and Pam3Cys in the presence or absence of the recombinant TirEs and $\mathrm{Hp} 1$, which was added to the cells before stimulation. IL- 8 was measured in the supernatant through ELISA. TirE1 and TirE2 both inhibited Malp2-, Pam2Cys-, and Pam3Cys-induced TLR2 activation significantly (Figure $7(b)$ ). This effect was similar to the inhibition by SSL3, a well-known staphylococcal inhibitor of TLR2 signaling [75]. However, the combination of TirE1, Hp1, and TirE2 had no inhibitory effect on TLR2-mediated release of IL-8 (Figure 7(b)). 


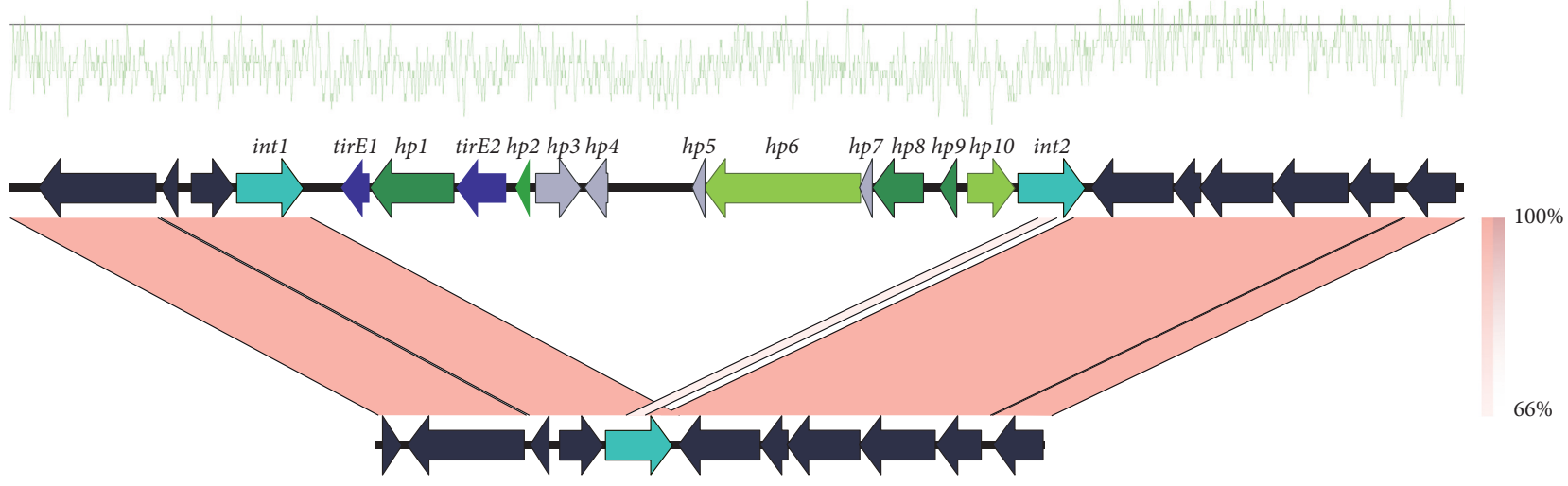

(a)

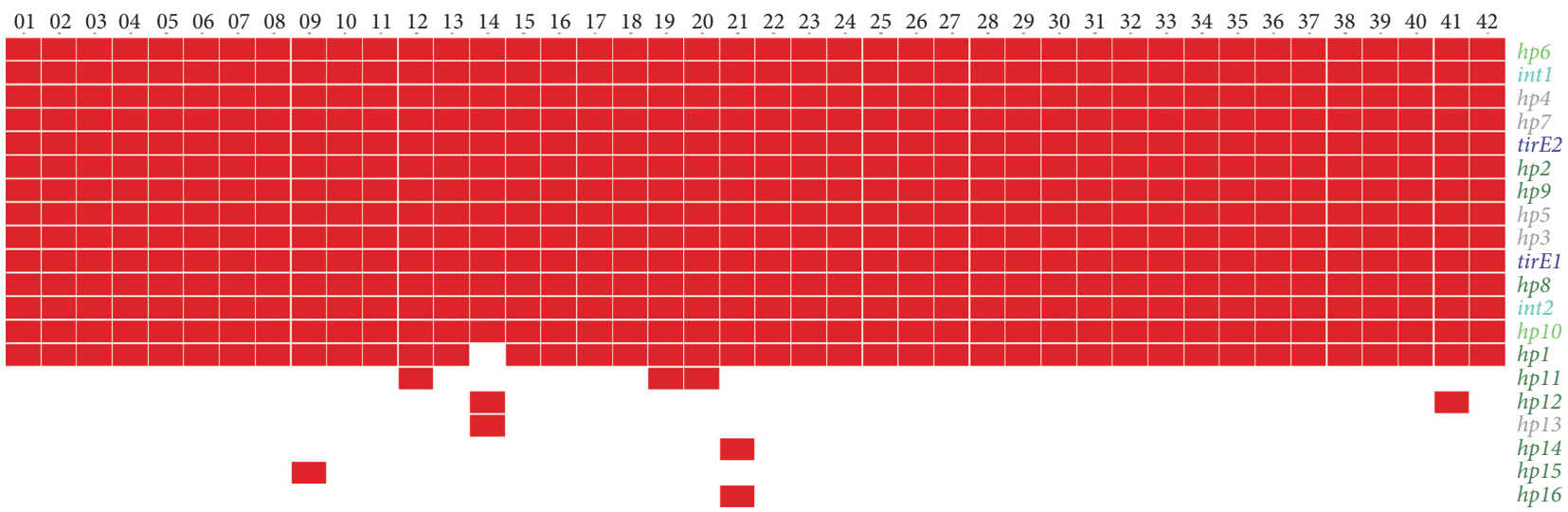

(b)

FIGURE 3: Comparative genomics of MGE-TirE insertion and relative abundance of MGE genes. (a) Pairwise alignment of E1162, containing MGE-TirE (top), and 17OM39. MGE-TirE is flanked by two integrases (cyan) and contains in total 14 open reading frames, color-coded by putative function. tirEs are shown in blue, putative DNA-binding hypothetical proteins in dark green, and putative transcription regulators in light green, hypothetical proteins (hp) for which no function could be predicted in grey. On top of the alignment, the GC abundance is illustrated in green. The black line refers to $50 \%$ GC. The percentage of nucleotide identity in forward and reverse strands is represented in light orange and dark orange, respectively, as shown in the bottom right of the alignment. (b) Gene presence/absence within MGE-TirE in 42 strains (top). Genes are color coded by the putative function of the proteins they encode (the same color code as in (a)).

3.8. The tirE Locus Does Not Have an Effect on Phagocytosis or Bacterial Survival within Macrophages. Since phagocytosis of enterococci is serum dependent [81-83], the effect of the possession of the tirE locus on serum-induced phagocytosis was further examined. Hence, the GFP-expressing enterococci (GFP-E1162 and GFP-E1162 $\Delta$ tirE) were incubated for $1 \mathrm{~h}$ in human blood, before the level of fluorescent bacteria within PMNs was analyzed by flow cytometry. There was no significant difference in the level of phagocytosis of the wildtype strain and its isogenic mutant (Figure S5A). The experiment was then repeated using blood cells. Here, GFP-E1162 and GFP-E1162 $\Delta$ tirE were opsonized by adding $50 \%$ or $100 \%$ serum before being exposed to human PMNs and PBMCs. Again, there was no significant difference between the phagocytosis of GFPE1162 and GFP-E1162 2 tirE (Figure S5B). This clearly shows that the tirE locus does not influence the hostmediated phagocytosis of the bacteria.

Next, the role of the tirE locus for intracellular survival in macrophages was investigated. E1162 and E1162 $\Delta$ tirE enterococci were coincubated with Thp-1 over time, before being plated out for CFU determination. $0.5 \%$ of E1162 and E1162 $\Delta$ tirE survived after $24 \mathrm{~h}$, and after $120 \mathrm{~h}$, very few bacteria had survived. The presence or absence of the tirE locus did not have any effect on the ability of the bacteria to survive in macrophages (Figure S6).

3.9. Presence of the tirE Locus Enhances Bacterial Survival and Proliferation in Blood. Finally, it was evaluated whether the presence of the tirE locus plays a role in E. faecium's survival in blood. E1162 and E1162 $\operatorname{tirE}$ were incubated in human whole blood for $3 \mathrm{~h}$ before plating for CFU determination. The wildtype strain showed a 19 -fold increase in CFU over the blood incubation, while its isogenic mutants showed only a 13-fold increase in CFU (Figure 8(a)). Additionally, the differences in survival and replication between E1162 and E1162 $\Delta$ tirE increased over time (up to $6 \mathrm{~h}$ ) (Figure 8(b)). This suggests that the presence of the tirE locus is beneficial for bacterial growth in human blood. 


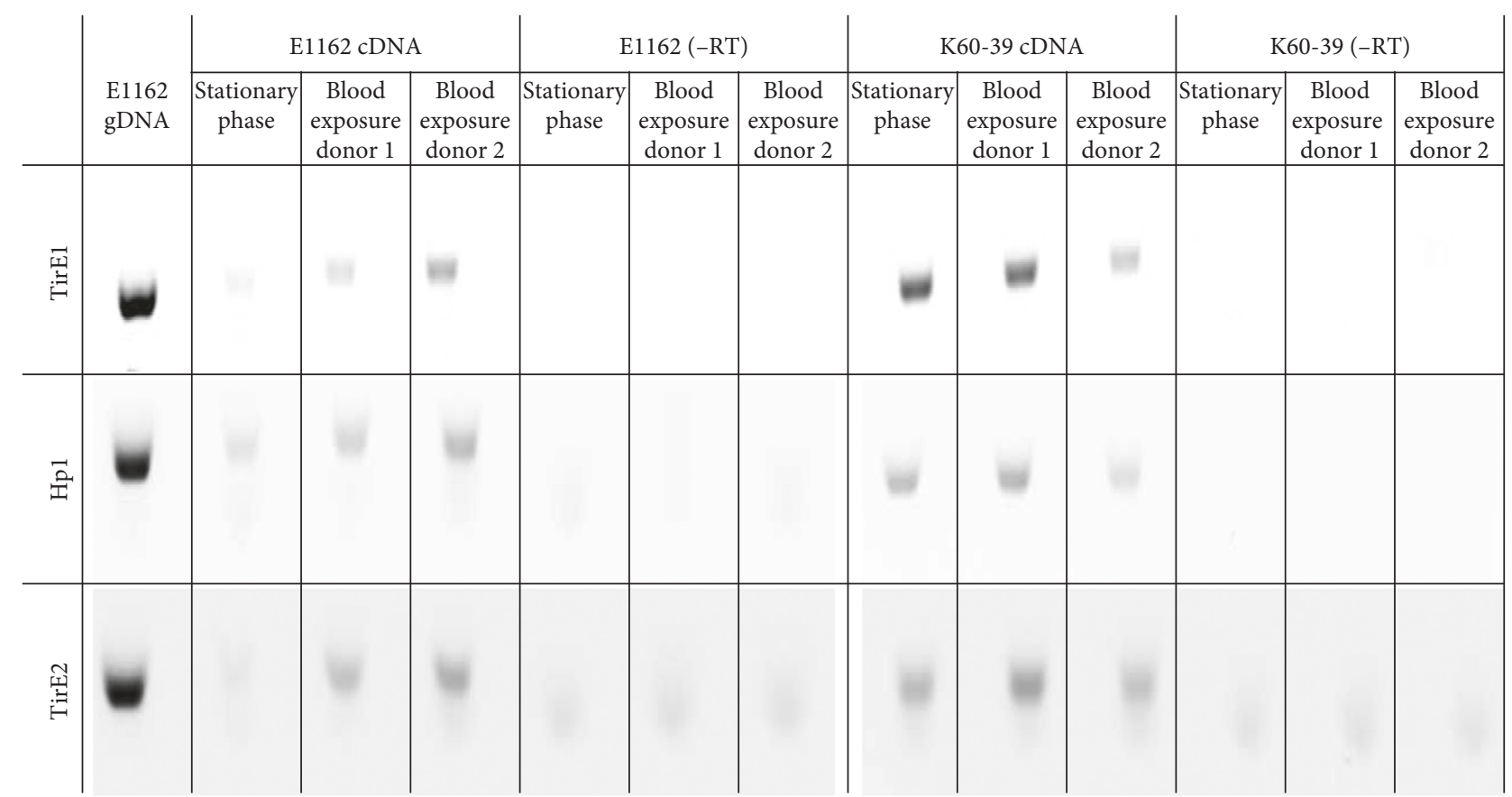

FIGURE 4: Expression of genes tirE1, hp1, and tirE2 upon exposure of E. faecium to blood. RT-PCR products of E1162 and K60-39 grown to the stationary phase in $\mathrm{BHI}$ and upon $3 \mathrm{~h}$ exposure to human blood of two different donors are photographed. -RT shows the control of the PCR reaction without reverse transcriptase.

\section{Discussion}

The pronounced increase in bacteremia caused by E. faecium $[7,84]$ and the limited knowledge about enterococcal virulence factors led to the search for TIR-domain-containing proteins in this species. This study identified a tirE locus encoding two TirE proteins and an intermediate protein Hp1. The tirE locus was prevalent in nosocomial isolates but absent in commensals (Figure 2) and located on a putative mobile genetic element of phage origin (Figure 3 ). The three genes may be expressed as an operon (Figure 5), and presence of all three proteins was confirmed in the bacterial cell lysate and supernatant (Figure 6). Moreover, the tirElocus genes were expressed when bacteria were exposed to blood (Figure 4), and the presence of the tirE locus increased bacterial proliferation in blood (Figure 8).

Hp1 intersected the two genes encoding the two TirE proteins presented here. Since we were able to link two of these genes by RT-PCR and no strong promoter was identified between the genes, we suggest that they are expressed as one operon. The tirE locus was found on a putative mobile genetic element. The element is putatively of phage origin because of its low GC content and the presence of phage proteins. Likewise, $t c p F$ of $E$. faecalis is found in a hotspot of recombination events of phage origin [24]. In fact, it has been observed multiple times that bacterial tir genes are found in regions of phage origin, e.g., $t p A$ (S. enterica) [18], tcpC (E. coli) and tcpB (Brucella) [85], or on mobile elements such as tirS (S. aureus) on SCC [86]. Thus, lateral transfer of bacterial tir genes is likely. Furthermore, the putative MGE-TirE appeared in multiple branches within the E. faecium phylogenetic tree (Figure S3), indicating horizontal gene transfer. However, it cannot be concluded whether the element was acquired or lost several times.

A high prevalence of the tirE locus was found in clinical and blood culture isolates, whereas the genes are absent in community isolates (Figure 2). Similar observations were made for $t c p C$, which was present in $40 \%$ of uropathogenic strains versus $8 \%$ in commensal strains [85]. TcpF is predominant among urinary tract infection isolates but also frequent in commensal isolates (76\% in invasive versus $58 \%$ in commensal strains) [21]. This could however be explained by the differences in the clade structure of E. faecium and E. faecalis: while E. faecium is characterized by host-specific lineages and clades, with a clearly distinct clade (A1) of isolates originating from hospitalized patients, this divergence is not seen in E. faecalis $[1,87]$. In this context, it makes sense that $E$. faecium tirE is not found in community isolates, while E. faecalis tcpF is. For E. faecalis, it was suggested that the possession of the $t c p F$ gene is also beneficial in community isolates, since the homeostasis in the gastrointestinal tract is crucial for commensals. Accordingly, avoiding NFkB activation through TLR signaling inhibition reduces the risk of homeostatic imbalance [21]. Besides the high prevalence of the tirE-locus genes in clinical and blood culture, it was observed that $10 \%$ of the hospital screening samples positive for tirE1 and tirE2, but lacking $h p 1$, were also positive for the $v a n B$ vancomycin-resistant cluster (Table S2). hp1, alias HMPREF0351_10592, was previously described as an insertion site for the conjugative vanB transposon Tn1549/5382 in different strain backgrounds (ST192, ST78, ST927, and ST202 isolates) [88]. This vanB transposon has also been shown to prefer AT-rich sequences 


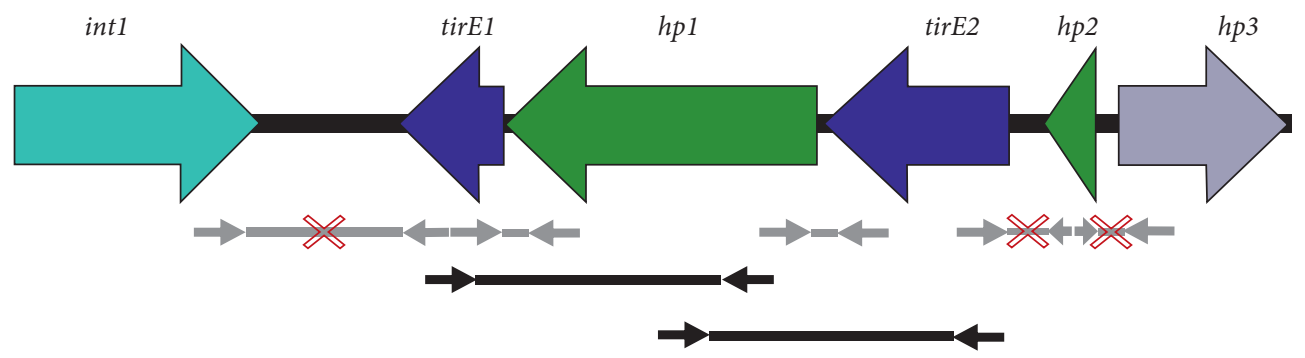

(a)

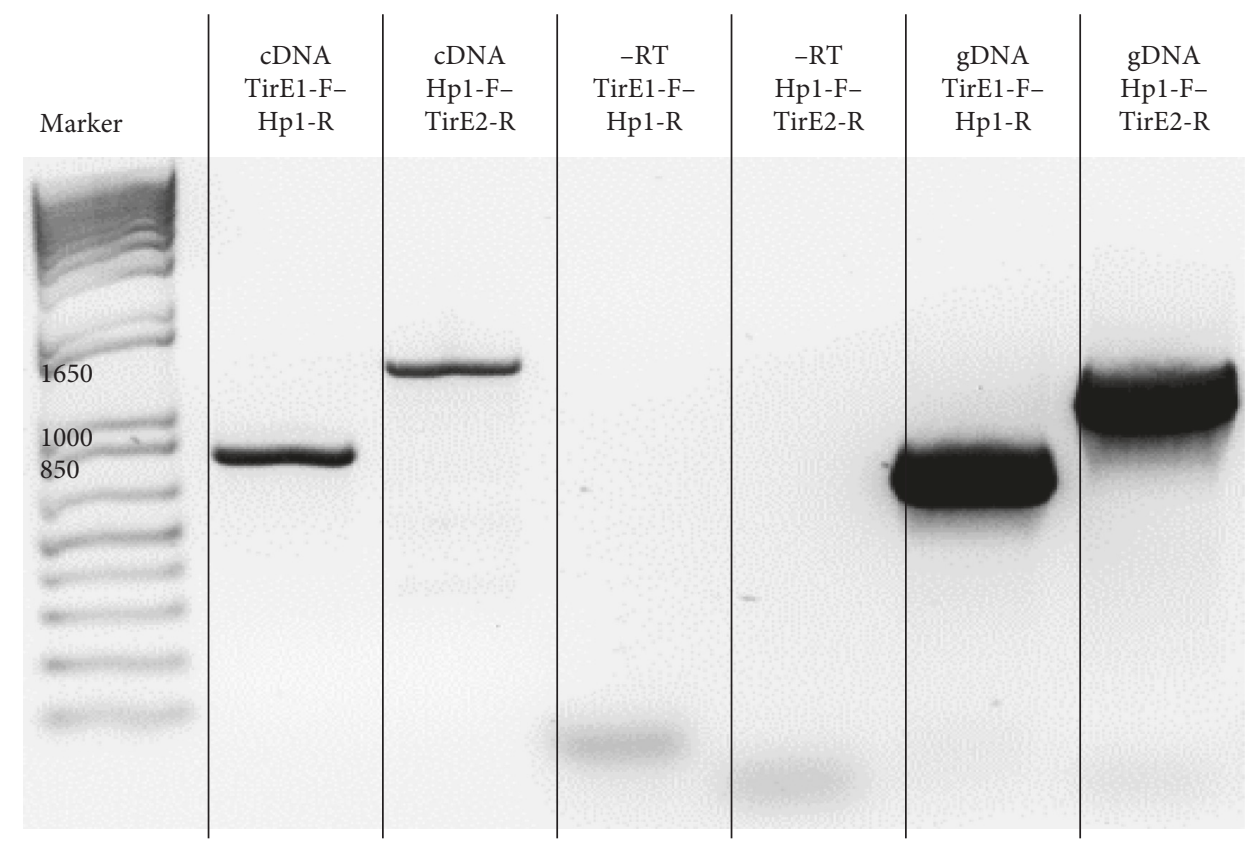

(b)

FIGURE 5: Both tirEs are part of one operon within E1162. (a) Graphical representation of the tirE operon within E1162 and primer locations. The ORFs in the immediate surrounding of tirEs are shown by thick arrows, while thin grey and black arrows indicate the location and orientation of the primers used for RT-PCR. Amplification is indicated as a straight line, and crossed lines indicate absence of products. Grey lines and arrows illustrate junction primers amplifying intergenic regions and their products. Black lines and arrows show the internal primers and their products, which were used to confirm linkage of the gene expression. (b) RT-PCR products linking tirE1, $h p 1$, and tirE2. RT-PCR after amplification with tirE1 internal (TirE1-F) plus $h p 1$ internal (Hp-R) primers yielded a 777 bp band and with $h p 1$ internal (Hp-F) plus tirE2 internal (TirE2-R) gave a 1286 bp product. -RT shows corresponding negative controls and gDNA positive controls.

[89], and the putative MGE-TirE with its $31 \%$ GC content is AT enriched. However, it remains to be investigated by future studies whether $h p 1$ provided an insertion site for the $v a n B$ transposon in our strain collection.

No direct bacterial contact with the host cell is needed to exert the function of bacterial TIR-domain-containing proteins, e.g., for E. coli TcpC [90] and S. aureus TirS [19], and bacterial TIR-domain-containing proteins have been detected inside host cells previously [85]. However, most of the bacterial TIR-domain-containing proteins lack secretion signals [22], and how the proteins are released and transferred to other cells remains largely unknown. Here, we detected all tirE-locus-encoded proteins in the bacterial supernatant and demonstrated that TirE2 and Hp1 can be found associated with bacterial MVs. Similarly, S. aureus TirS was also associated with MVs isolated from bacteria grown in BHI (results not shown). This might suggest that bacterial TIR-domain-containing proteins can be released through MVs. In order to test this hypothesis, the vesicular proteomic contents of other tir-gene-possessing bacteria should be evaluated and compared to the nonmembrane bound secretome.

This study found that purified TirEs negatively interfere with IL-8 release of HEK293T cells expressing TLR2 when added to the cell cultures prior to stimulation with its specific ligands (Figure 7(b)). These findings are in line with results from NFkB-reporter assays for E. faecalis TcpF, conducted in hTLR2-expressing HEK293 cells stimulated with PamCys3 [21], and with similar studies on S. aureus TirS $[19,86]$. Bacterial TIR-domain-containing proteins from Gramnegative bacteria $[18,22,90]$ have also been shown to impede the host innate immune response through interference 


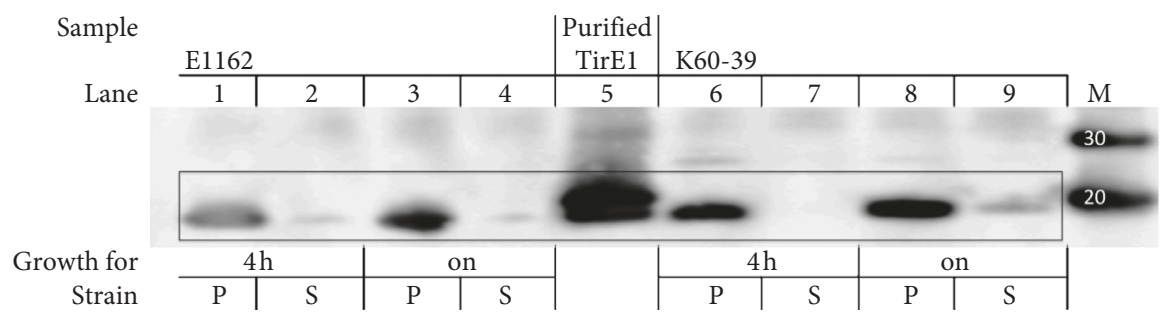

(a)

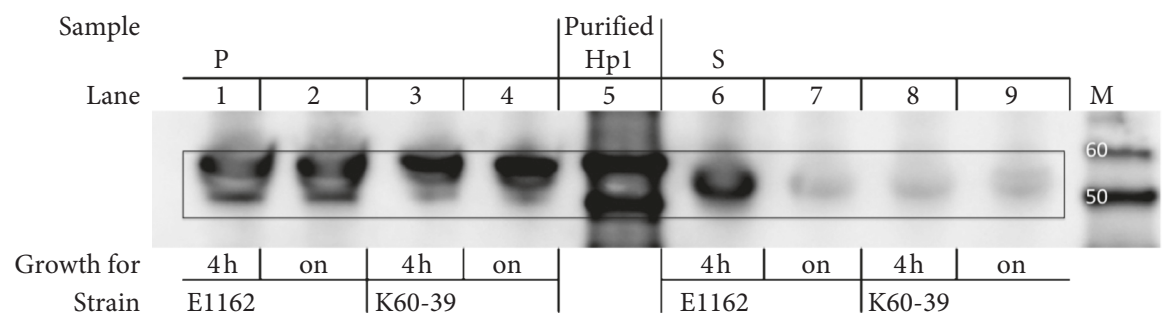

(b)

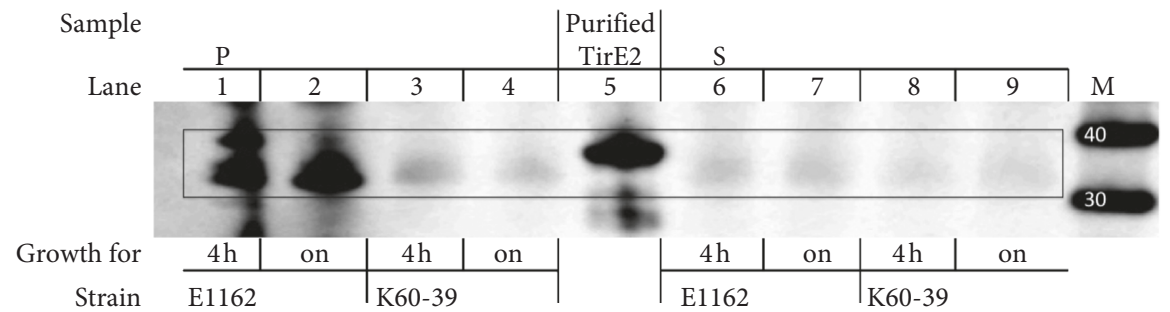

(c)

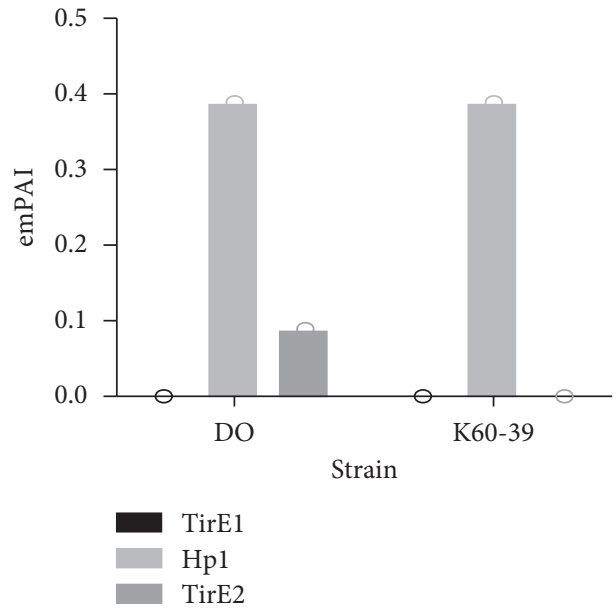

(d)

Figure 6: Detection of TirEs and Hp1 in the bacterial cell lysate, supernatant, and MV. (a-c) TirE1 (18.5 kDa), Hp1 (55 kDa), and TirE2 $(33 \mathrm{kDa})$ were immunoprecipitated from the lysed pellet $(\mathrm{P})$ or bacterial supernatant $(\mathrm{S})$ after $4 \mathrm{~h}$ or from overnight culture (on) and detected in western blot with anti-TirE1, anti-Hp1, or anti-TirE2 serum. Shown are samples from the E1162 and K60-39, purified proteins (TirE1 with his tag $20.4 \mathrm{kDa}$ (lane 5 in (a)), Hp1 with his tag $57 \mathrm{kDa}$ (lane $5 \mathrm{in}$ (b)), and TirE2 with his tag $35 \mathrm{kDa}$ (lane 5 in (c))), and Magic marker $(\mathrm{M})$ with size indicated in $\mathrm{kDa}$. (d) EmPAI values indicating the protein abundance of TirE1, Hp1, and TirE2 proteins in membrane vesicles derived from K60-39 and DO.

with TLR signaling. The inhibitory effect of TirEs on IL-8 release was abolished when a combination of TirEs and Hp1 was used. Similarly, there was no difference in cytokine release in macrophage-like cells infected with E1162 or E1162 $\Delta$ tirE (Figure 7(a)). The mechanism behind this phenomenon is elusive but might involve a regulatory role of
Hp1. Unfortunately, it was not possible to make single knockouts to distinguish between effects of the proteins. Two staphylococcal TIR-domain-containing proteins, SaTlp1 and SaTlp2, found in zoonotic ST398, also showed a deviation from the typical theme: they upregulated NFkB signaling instead of downregulating it [20]. 


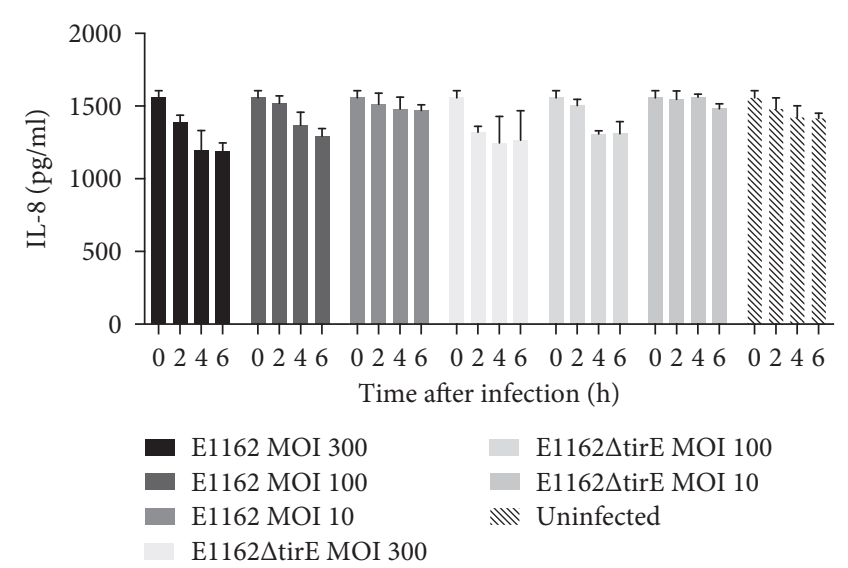

(a)

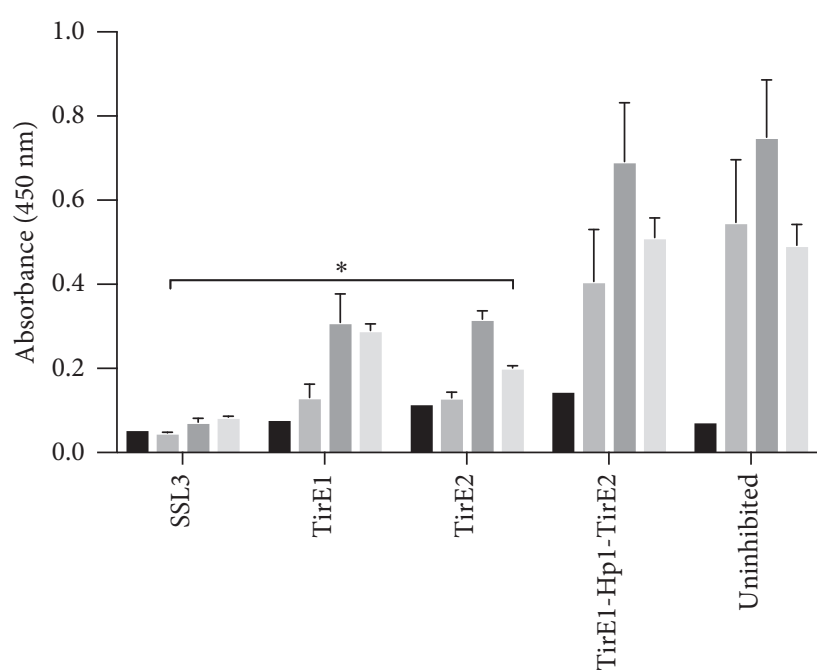

\begin{tabular}{l|l} 
Nonstimulated & Pam2 \\
Malp2 & Pam3
\end{tabular}

(b)

Figure 7: Effect of TirEs and Hp1 on IL-8 release. (a) Presence of tirE1-hp1-tirE2 does not affect IL-8 release. Upon infection of macrophagelike cells (differentiated Thp1 cells) with E1162 and E1162 $\Delta$ tir at MOI 300, MOI 100, and MOI 10, IL-8 was measured in the cellular supernatant at $2 \mathrm{~h}, 4 \mathrm{~h}$, and $6 \mathrm{~h}$ after infection, through IL-8 ELISA. Data are pooled from 3 independent experiments, each in duplicates. (b) Interference of TirE1 and TirE2 with TLR2 signaling. IL-8 release was measured as a response of HEK293 cells stably expressing TLR2 towards stimulation with the lipoproteins Malp2, Pam2Cys, and Pam3Cys. The cells were left untreated (nonstimulated) or were added Malp2, Pam2Cys, or Pam3Cys. One hour before stimulation with agonists, the TLR2-expressing HEK293 cells were added SSL3 (wellknown inhibitor of TLR2 signaling), TirE1, TirE2, or a combination of TirE1, Hp1, and TirE2 to a final concentration of $10 \mu \mathrm{g} / \mathrm{ml}$. IL-8 was quantified in IL-8 ELISA, using TMB as the substrate and measuring the absorbance at $450 \mathrm{~nm}$. Data are shown in triplicates, representative of 3 independent experiments. ${ }^{*} P \leq 0.05$, inhibited vs uninhibited: $P_{\text {Tir1Malp2 }}=0.051, P_{\text {Tir1Pam } 2}=0.045, P_{\text {Tir1Pam3 }}=0.017, P_{\text {Tir2Malp2 }}=0.049$, $P_{\text {Tir2Pam } 2}=0.035$, and $P_{\text {Tir2Pam3 }}=0.004 ;$ Abs: absorption.

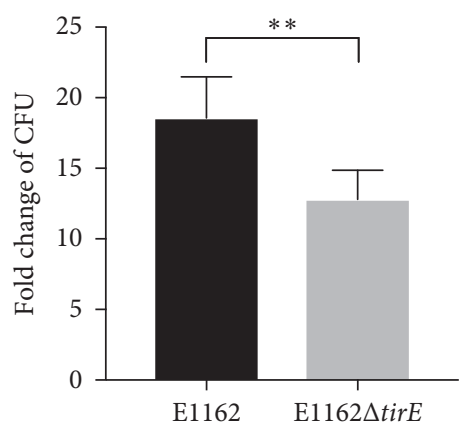

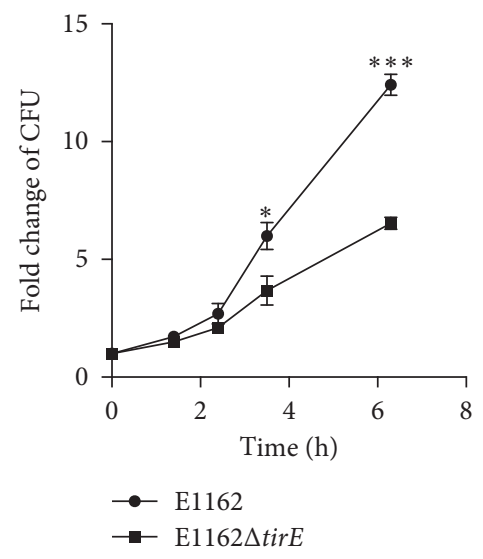

(b)

Figure 8: Presence of tirE1-hp1-tirE2 increases bacterial survival and proliferation in human whole blood ex vivo. (a) tirE1-hp1-tirE2 enhances bacterial proliferation in human whole blood. After $3 \mathrm{~h}$ of exposure of E1162 and E1162 $\Delta$ tirE to whole blood, the fold change in $\mathrm{CFU}$ is significantly higher for E1162 compared to E1162 $\Delta$ tirE, where the inoculum is set as 1 . Data shown represent pooled data from 3 donors (inoculum $20 \mu \mathrm{l}$ at $\mathrm{OD}_{600} 0.4$, $t$-test, 2-tailed, $P_{3 h}=0.002$ ). (b) tirE1-hp1-tirE2 enhances proliferation in human whole blood over time. E1162 and E1162 $\Delta$ tirE were incubated in blood for indicated time points. Data represent quadruplicates from one healthy donor and are representative of 3 independent experiments in different donors. (inoculum $=20 \mu \mathrm{l}$ at $\mathrm{OD}_{600} 0.4, t$-test, $P_{3.5 h}=0.0328, P_{6.3 h}<0.0001$ ). 
In E. faecium, the tirE locus promoted bacterial proliferation in human blood (Figure 8) and blood also induced tirE expression (Figure 4). This suggests that the tirE locus provides E. faecium with an advantage of surviving in blood by interfering with TLR-2 signaling. This could also explain the predominance of tirE and $h p 1$ in blood culture and hospital isolates.

\section{Conclusion}

The presented study describes TirEs, E. faecium TIRdomain-containing proteins, as novel virulence factors. The tirE locus is exclusive of E. faecium nosocomial isolates and located on a putative mobile genetic element of phage origin. Bacterial proliferation within human blood is enhanced by possession of the tirE locus, implicating a role of TirEs in bacteremia and the pathogenicity of E. faecium.

\section{Data Availability}

The datasets analyzed during the current study are included in the supplementary material file and are available from the corresponding author upon reasonable request.

\section{Conflicts of Interest}

The authors declare that the research was conducted in the absence of any commercial or financial relationships that could be construed as potential conflicts of interest.

\section{Authors' Contributions}

Mona Johannessen and Kristin Hegstad contributed equally to this work.

\section{Acknowledgments}

We thank A. Didriksen, A. Mekhlif, P. Aerts, and K. Julin for excellent technical assistance. This study was funded by the Northern Norway Regional Health Authority Medical Research Program (SFP1157-14 and SFP1231-15) and by travel grants from Research School NFR project number 249062. The publication charges for this article have been funded by a grant from the publication fund of UiT-The Arctic University of Norway.

\section{Supplementary Materials}

Figure S1: expression and purification of TirE1 and TirE2. Figure S2: phylogenetic tree of eukaryotic and prokaryotic TIR-domain-containing proteins. Figure S3: midpoint rooted tree displaying the presence and absence of tirE1$h p 1$-tirE2. Figure S4: confirmation of successful construction of E1162 $\Delta$ tirE. Figure S5: tirE1-hp1-tirE2 does not influence the immune cells ability to phagocytose the bacteria. Figure S6: presence of tirE1-hp1-tirE2 does not influence bacterial survival within macrophages. Table S1: prevalence of the tirE locus. Table S2: strains and plasmids used for laboratory experiments. Table S3: primers used in the study. Table S4: metadata of E. faecium genomes harboring tirE1-hp1-tirE2 genes. (Supplementary Materials)

\section{References}

[1] A. M. Guzman Prieto, W. van Schaik, M. R. C. Rogers et al., "Global emergence and dissemination of enterococci as nosocomial pathogens: attack of the clones?," Frontiers in Microbiology, vol. 7, p. 788, 2016.

[2] C. A. Arias and B. E. Murray, "The rise of the Enterococcus: beyond vancomycin resistance," Nature Reviews Microbiology, vol. 10, no. 4, pp. 266-278, 2012.

[3] M. S. Gilmore, F. Lebreton, and W. van Schaik, "Genomic transition of enterococci from gut commensals to leading causes of multidrug-resistant hospital infection in the antibiotic era," Current Opinion in Microbiology, vol. 16, no. 1, pp. 10-16, 2013.

[4] N. I. A. Higuita and M. M. Huycke, Enterococcal Disease, Epidemiology, and Implications for Treatment, Massachusetts Eye and Ear Infirmary, Boston, MA, USA, 2014, https:// www.ncbi.nlm.nih.gov/books/NBK190429/.

[5] T. Raza, S. R. Ullah, K. Mehmood, and S. Andleeb, "Vancomycin resistant enterococci: a brief review," Journal of Pakistan Medical Association, vol. 68, pp. 768-772, 2018.

[6] A. N. Treitman, P. R. Yarnold, J. Warren, and G. A. Noskin, "Emerging incidence of Enterococcus faecium among hospital isolates (1993 to 2002)," Journal of Clinical Microbiology, vol. 43, no. 1, pp. 462-463, 2005.

[7] M. E. A. de Kraker, V. Jarlier, J. C. M. Monen, O. E. Heuer, N. van de Sande, and H. Grundmann, "The changing epidemiology of bacteraemias in europe: trends from the european antimicrobial resistance surveillance system," Clinical Microbiology and Infection, vol. 19, no. 9, pp. 860-868, 2013.

[8] F. Lebreton, W. van Schaik, A. M. McGuire et al., "Emergence of epidemic multidrug-resistant Enterococcus faecium from animal and commensal strains," mBio, vol. 4, no. 4, pp. e00534-13, 2013.

[9] F. Lebreton, A. L. Manson, J. T. Saavedra, T. J. Straub, A. M. Earl, and M. S. Gilmore, "Tracing the enterococci from paleozoic origins to the hospital," Cell, vol. 169, no. 5, pp. 849-861, 2017.

[10] R. Medzhitov, "Toll-like receptors and innate immunity," Nature Reviews Immunology, vol. 1, no. 2, pp. 135-145, 2001.

[11] F. Askarian, T. Wagner, M. Johannessen, and V. Nizet, "Staphylococcus aureus modulation of innate immune responses through Toll-like (TLR), (NOD)-like (NLR) and C-type lectin (CLR) receptors," FEMS Microbiology Reviews, vol. 42 , no. $5,2018$.

[12] M. Leendertse, R. J. L. Willems, I. A. J. Giebelen et al., “TLR2dependent MyD88 signaling contributes to early host defense in murine Enterococcus faecium peritonitis," Journal of Immunology, vol. 180, no. 7, pp. 4865-4874, 2008.

[13] T. Kawai, O. Adachi, T. Ogawa, K. Takeda, and S. Akira, "Unresponsiveness of MyD88-deficient mice to endotoxin," Immunity, vol. 11, no. 1, pp. 115-122, 1999.

[14] O. Adachi, T. Kawai, K. Takeda et al., "Targeted disruption of the MyD88 gene results in loss of IL-1- and IL-18-mediated function," Immunity, vol. 9, no. 1, pp. 143-150, 1998.

[15] S. Pandey, T. Kawai, and S. Akira, "Microbial sensing by tolllike receptors and intracellular nucleic acid sensors," Cold Spring Harbor Perspectives in Biology, vol. 7, no. 1, 2015.

[16] C. E. Stebbins and J. E. Galán, "Structural mimicry in bacterial virulence,” Nature, vol. 412, no. 6848, pp. 701-705, 2001. 
[17] S. L. Chan, L. Y. Low, S. Hsu et al., "Molecular mimicry in innate immunity: crystal structure of a bacterial TIR domain," Journal of Biological Chemistry, vol. 284, no. 32, pp. 2138621392, 2009.

[18] R. M. Newman, P. Salunkhe, A. Godzik, and J. C. Reed, "Identification and characterization of a novel bacterial virulence factor that shares homology with mammalian toll/ interleukin-1 receptor family proteins," Infection and Immunity, vol. 74, no. 1, pp. 594-601, 2006.

[19] F. Askarian, N. M. van Sorge, M. Sangvik et al., "A Staphylococcus aureus TIR domain protein virulence factor blocks TLR2-mediated NF- $\mathrm{B}$ signaling," Journal of Innate Immunity, vol. 6, no. 4, pp. 485-498, 2014.

[20] N. J. Patterson, J. Günther, A. J. Gibson et al., "Two TIR-like domain containing proteins in a newly emerging zoonotic Staphylococcus aureus strain sequence type 398 are potential virulence factors by impacting on the host innate immune response," Frontiers in Microbiology, vol. 5, 2014.

[21] T. D. Kraemer, O. D. Quintanar Haro, E. Domann, T. Chakraborty, and S. Tchatalbachev, "The TIR domain containing locus of Enterococcus faecalis is predominant among urinary tract infection isolates and downregulates host inflammatory response," International Journal of Microbiology, vol. 2014, Article ID 918143, 9 pages, 2014.

[22] R. R. Rana, M. Zhang, A. M. Spear, H. S. Atkins, and B. Byrne, "Bacterial TIR-containing proteins and host innate immune system evasion," Medical Microbiology and Immunology, vol. 202, no. 1, pp. 1-10, 2013.

[23] J. L. Slack, K. Schooley, T. P. Bonnert et al., "Identification of two major sites in the type I interleukin-1 receptor cytoplasmic region responsible for coupling to pro-inflammatory signaling pathways," Journal of Biological Chemistry, vol. 275, no. 7 , pp. $4670-4678,2000$.

[24] J. Zou, A. S. Baghdayan, S. J. Payne, and N. Shankar, "A TIR domain protein from $E$. faecalis attenuates MyD88-mediated signaling and NF- $\mathrm{BB}$ activation," PloS One, vol. 9, no. 11, Article ID e112010, 2014.

[25] T. J. Eaton and M. J. Gasson, "Molecular screening of Enterococcus virulence determinants and potential for genetic exchange between food and medical isolates," Applied and Environmental Microbiology, vol. 67, no. 4, pp. 1628-1635, 2001.

[26] S. R. Nallapareddy, K. V. Singh, and B. E. Murray, "Construction of improved temperature-sensitive and mobilizable vectors and their use for constructing mutations in the adhesin-encoding acm gene of poorly transformable clinical Enterococcus faecium strains," Applied and Environmental Microbiology, vol. 72, no. 1, pp. 334-345, 2006.

[27] E. Heikens, M. J. M. Bonten, and R. J. L. Willems, "Enterococcal surface protein Esp is important for biofilm formation of Enterococcus faecium E1162," Journal of Bacteriology, vol. 189, no. 22, pp. 8233-8240, 2007.

[28] J. Sillanpää, S. R. Nallapareddy, V. P. Prakash et al., "Identification and phenotypic characterization of a second collagen adhesin, Scm, and genome-based identification and analysis of 13 other predicted MSCRAMMs, including four distinct pilus loci, in Enterococcus faecium," Microbiology, vol. 154, no. 10, pp. 3199-3211, 2008.

[29] A. P. A. Hendrickx, M. J. M. Bonten, M. van Luit-Asbroek, C. M. E. Schapendonk, A. H. M. Kragten, and R. J. L. Willems, "Expression of two distinct types of pili by a hospital-acquired Enterococcus faecium isolate," Microbiology, vol. 154, no. 10, pp. 3212-3223, 2008.
[30] A. P. A. Hendrickx, M. van Luit-Asbroek, C. M. E. Schapendonk et al., "SgrA, a nidogen-binding LPXTG surface adhesin implicated in biofilm formation, and EcbA, a collagen binding MSCRAMM, are two novel adhesins of hospital-acquired Enterococcus faecium," Infection and Immunity, vol. 77, no. 11, pp. 5097-5106, 2009.

[31] J. Sillanpää, S. R. Nallapareddy, K. V. Singh et al., "Characterization of the ebpfm pilus-encoding operon of Enterococcus faecium and its role in biofilm formation and virulence in a murine model of urinary tract infection," Virulence, vol. 1, no. 4, pp. 236-246, 2010.

[32] J. J. Johanson, L. Feriancikova, and S. Xu, "Influence of enterococcal surface protein (esp) on the transport of Enterococcus faecium within saturated quartz sands," Environmental Science and Technology, vol. 46, no. 3, pp. 1511-1518, 2012.

[33] F. L. Paganelli, R. J. L. Willems, P. Jansen et al., "Enterococcus faecium biofilm formation: identification of major autolysin AtlAEfm, associated acm surface localization, and AtlAEfmindependent extracellular DNA release," mBio, vol. 4, no. 2, 2013.

[34] P. Gao, K. L. Pinkston, A. Bourgogne et al., "Library screen identifies Enterococcus faecalis CcpA, the catabolite control protein A, as an effector of Ace, a collagen adhesion protein linked to virulence," Journal of Bacteriology, vol. 195, no. 20, pp. 4761-4768, 2013.

[35] X. Zhang, J. Top, M. de Been et al., "Identification of a genetic determinant in clinical Enterococcus faecium strains that contributes to intestinal colonization during antibiotic treatment," Journal of Infectious Diseases, vol. 207, no. 11, pp. 1780-1786, 2013.

[36] S. R. Somarajan, J. H. Roh, K. V. Singh, G. M. Weinstock, and B. E. Murray, "CcpA is important for growth and virulence of Enterococcus faecium," Infection and Immunity, vol. 82, no. 9, pp. 3580-3587, 2014.

[37] S. R. Somarajan, S. L. La Rosa, K. V. Singh, J. H. Roh, M. Höök, and B. E. Murray, “The fibronectin-binding protein Fnm contributes to adherence to extracellular matrix components and virulence of Enterococcus faecium," Infection and Immunity, vol. 83, no. 12, pp. 4653-4661, 2015.

[38] A. M. G. Prieto, R. T. Urbanus, X. Zhang et al., "The $\mathrm{N}$-terminal domain of the thermo-regulated surface protein PrpA of Enterococcus faecium binds to fibrinogen, fibronectin and platelets," Scientific Reports, vol. 5, no. 1, 2015.

[39] F. L. Paganelli, M. de Been, J. C. Braat et al., "Distinct SagA from hospital-associated clade A1 Enterococcus faecium strains contributes to biofilm formation," Applied and Environmental Microbiology, vol. 81, no. 19, pp. 6873-6882, 2015.

[40] F. L. Paganelli, J. Huebner, K. V. Singh et al., "Genome-wide screening identifies phosphotransferase system permease BepA to Be involved in Enterococcus faecium endocarditis and biofilm formation," Journal of Infectious Diseases, vol. 214, no. 2, pp. 189-195, 2016.

[41] L. Ali, M. Spiess, D. Wobser, M. Rodriguez, H. E. Blum, and T. Sakınç, "Identification and functional characterization of the putative polysaccharide biosynthesis protein (CapD) of Enterococcus faecium U0317," Infection, Genetics and Evolution, vol. 37, pp. 215-224, 2016.

[42] W. Gao, B. P. Howden, and T. P. Stinear, "Evolution of virulence in Enterococcus faecium, a hospital-adapted opportunistic pathogen," Current Opinion in Microbiology, vol. 41, pp. 76-82, 2018. 
[43] A. Marchler-Bauer, M. K. Derbyshire, N. R. Gonzales et al., "CDD: NCBI's conserved domain database," Nucleic Acids Res, vol. 43, no. D1, pp. D222-226, 2015.

[44] K. Katoh and D. M. Standley, "MAFFT multiple sequence alignment software version 7: improvements in performance and usability," Molecular Biology and Evolution, vol. 30, no. 4, pp. 772-780, 2013.

[45] A. Larsson, "AliView: a fast and lightweight alignment viewer and editor for large datasets," Bioinformatics, vol. 30, no. 22, pp. 3276-3278, 2014.

[46] B. Kaplan-Türköz, T. Koelblen, C. Felix et al., "Structure of the Toll/interleukin 1 receptor (TIR) domain of the immunosuppressive Brucella effector BtpA/Btp1/TcpB," FEBS Letters, vol. 587, no. 21, pp. 3412-3416, 2013.

[47] H. M. Berman, J. Westbrook, Z. Feng et al., "The protein data bank," Nucleic Acids Research, vol. 28, no. 1, pp. 235-242, 2000.

[48] RCSB PDB: Homepage. https://www.rcsb.org/.

[49] A. Stamatakis, "RAxML version 8: a tool for phylogenetic analysis and post-analysis of large phylogenies," Bioinformatics, vol. 30, no. 9, pp. 1312-1313, 2014.

[50] L. A. Kelley, S. Mezulis, C. M. Yates, M. N. Wass, and M. J. E. Sternberg, "The Phyre2 web portal for protein modeling, prediction and analysis," Nature Protocols, vol. 10, no. 6 , pp. 845-858, 2015.

[51] $\mathrm{PDBe}<$ Fold < EMBL-EBI. http://www.ebi.ac.uk/msd-srv/ ssm/cgi-bin/ssmserver.

[52] PyMOL, https://www.pymol.org/.

[53] S. F. Altschul, W. Gish, W. Miller, E. W. Myers, and D. J. Lipman, "Basic local alignment search tool," Journal of Molecular Biology, vol. 215, pp. 403-410, 1990.

[54] BLAST: Basic Local Alignment Search Tool. https:// blast.ncbi.nlm.nih.gov/Blast.cgi.

[55] T. J. Treangen, B. D. Ondov, S. Koren, and A. M. Phillippy, "The Harvest suite for rapid core-genome alignment and visualization of thousands of intraspecific microbial genomes," Genome Biology, vol. 15, no. 11, p. 524, 2014.

[56] FigTree. http://tree.bio.ed.ac.uk/software/figtree/.

[57] A. R. Wattam, D. Abraham, O. Dalay et al., "PATRIC, the bacterial bioinformatics database and analysis resource," Nucleic Acids Research, vol. 42, no. D1, pp. D581-591, 2014.

[58] T. J. Carver, K. M. Rutherford, M. Berriman, M.-A. Rajandream, B. G. Barrell, and J. Parkhill, "ACT: the Artemis comparison tool," Bioinformatics, vol. 21, no. 16, pp. 3422-3423, 2005.

[59] M. J. Sullivan, N. K. Petty, and S. A. Beatson, "Easyfig: a genome comparison visualizer," Bioinformatics, vol. 27 , no. 7 , pp. 1009-1010, 2011.

[60] Easyfig-home. http://mjsull.github.io/Easyfig/.

[61] T. Seemann, "Prokka: rapid prokaryotic genome annotation," Bioinformatics, vol. 30, no. 14, pp. 2068-2069, 2014.

[62] A. J. Page, C. A. Cummins, M. Hunt et al., "Roary: rapid largescale prokaryote pan genome analysis," Bioinformatics, vol. 31, no. 22, pp. 3691-3693, 2015.

[63] C. Perez-Llamas and N. Lopez-Bigas, "Gitools: analysis and visualisation of genomic data using interactive heat-maps," PLoS ONE, vol. 6, no. 5, 2011.

[64] Gitools-A Framework for Analysis and Visualization of Genomic Data. http://www.gitools.org/.

[65] J. Bestebroer, M. J. J. G. Poppelier, L. H. Ulfman et al., "Staphylococcal superantigen-like 5 binds PSGL-1 and inhibits P-selectin-mediated neutrophil rolling," Blood, vol. 109, no. 20, pp. 2936-2943, 2007.
[66] M. Johannessen, M. P. Delghandi, A. Rykx et al., "Protein kinase $\mathrm{D}$ induces transcription through direct phosphorylation of the cAMP-response element-binding protein," Journal of Biological Chemistry, vol. 282, no. 20, pp. 14777-14787, 2007.

[67] T. Wagner, B. Joshi, J. Janice et al., "Enterococcus faecium produces membrane vesicles containing virulence factors and antimicrobial resistance related proteins," Journal of Proteomics, vol. 187, pp. 28-38, 2018.

[68] B. Sauer, "Functional expression of the cre-lox site-specific recombination system in the yeast Saccharomyces cerevisiae," Molecular and Cellular Biology, vol. 7, no. 6, pp. 2087-2096, 1987.

[69] X. Zhang, F. L. Paganelli, D. Bierschenk et al., "Genome-Wide identification of ampicillin resistance determinants in Enterococcus faecium," PLoS Genetics, vol. 8, no. 6, article e1002804, 2012.

[70] X. Zhang, J. E. P. Vrijenhoek, M. J. M. Bonten, R. J. L. Willems, and W. van Schaik, "A genetic element present on megaplasmids allows Enterococcus faecium to use raffinose as carbon source," Environmental Microbiology, vol. 13, no. 2, pp. 518-528, 2011.

[71] A. McKenna, M. Hanna, E. Banks et al., "The Genome Analysis Toolkit: a MapReduce framework for analyzing nextgeneration DNA sequencing data," Genome Research, vol. 20, no. 9, pp. 1297-1303, 2010.

[72] GATK | Home. https://software.broadinstitute.org/gatk/.

[73] J. Top, (2018).

[74] K. J. Koymans, (2018).

[75] K. J. Koymans, L. J. Feitsma, T. H. C. Brondijk et al., "Structural basis for inhibition of TLR2 by staphylococcal superantigen-like protein 3 (SSL3)," Proceedings of the National Academy of Sciences, vol. 112, no. 35, pp. 11018-11023, 2015.

[76] C. Felix, B. Kaplan Türköz, S. Ranaldi et al., “The Brucella TIR domain containing proteins BtpA and BtpB have a structural WxxxE motif important for protection against microtubule depolymerisation," Cell Communication and Signaling, vol. 12, no. 1, p. 53, 2014.

[77] T. Ve, S. J. Williams, and B. Kobe, "Structure and function of Toll/interleukin-1 receptor/resistance protein (TIR) domains," Apoptosis, vol. 20, no. 2, pp. 250-261, 2015.

[78] X. Qin, J. R. Galloway-Peña, J. Sillanpaa et al., "Complete genome sequence of Enterococcus faecium strain TX16 and comparative genomic analysis of Enterococcus faecium genomes," BMC Microbiology, vol. 12, no. 1, p. 135, 2012.

[79] M. Ventura, C. Canchaya, M. Kleerebezem, W. M. de Vos, R. J. Siezen, and H. Brüssow, "The prophage sequences of Lactobacillus plantarum strain WCFS1," Virology, vol. 316, no. 2, pp. 245-255, 2003.

[80] L. Brown, J. M. Wolf, R. Prados-Rosales, and A. Casadevall, "Through the wall: extracellular vesicles in Gram-positive bacteria, mycobacteria and fungi," Nature Reviews Microbiology, vol. 13, no. 10, pp. 620-630, 2015.

[81] B. S. Harvey, C. J. Baker, and M. S. Edwards, "Contributions of complement and immunoglobulin to neutrophil-mediated killing of enterococci," Infection and Immunity, vol. 60, pp. 3635-3640, 1992.

[82] R. C. Arduino, B. E. Murray, and R. M. Rakita, "Roles of antibodies and complement in phagocytic killing of enterococci," Infection and Immunity, vol. 62, pp. 987-993, 1994.

[83] F. Romero-Saavedra, D. Laverde, D. Wobser et al., "Identification of peptidoglycan-associated proteins as vaccine 
candidates for enterococcal infections," PLoS ONE, vol. 9, no. 11, Article ID e111880, 2014.

[84] C. Prematunge, C. MacDougall, J. Johnstone et al., "VRE and VSE bacteremia outcomes in the era of effective VRE therapy: a systematic review and meta-analysis," Infection Control and Hospital Epidemiology, vol. 37, no. 1, pp. 26-35, 2016.

[85] C. Cirl, A. Wieser, M. Yadav et al., "Subversion of Toll-like receptor signaling by a unique family of bacterial Toll/ interleukin-1 receptor domain-containing proteins," Nature Medicine, vol. 14, no. 4, pp. 399-406, 2008.

[86] S. Patot, P. R. Imbert, J. Baude et al., "The TIR homologue lies near resistance genes in Staphylococcus aureus, coupling modulation of virulence and antimicrobial susceptibility," PLOS Pathogens, vol. 13, no. 1, article e1006092, 2017.

[87] E. B. Kim and M. L. Marco, "Nonclinical and clinical Enterococcus faecium strains, but not Enterococcus faecalis strains, have distinct structural and functional genomic features," Applied and Environmental Microbiology, vol. 80, no. 1, pp. 154-165, 2014.

[88] J. K. Bender, A. Kalmbach, C. Fleige, I. Klare, S. Fuchs, and G. Werner, "Population structure and acquisition of the vanB resistance determinant in German clinical isolates of Enterococcus faecium ST192," Scientific Reports, vol. 6, no. 1, 2016.

[89] L. Lambertsen, A. Rubio-Cosials, K. R. Patil, and O. Barabas, "Conjugative transposition of the vancomycin resistance carrying Tn1549: enzymatic requirements and target site preferences," Molecular Microbiology, vol. 107, no. 5, pp. 639-658, 2018.

[90] G. A. Snyder, C. Cirl, J. Jiang et al., "Molecular mechanisms for the subversion of MyD88 signaling by TcpC from virulent uropathogenic Escherichia coli," Proceedings of the National Academy of Sciences, vol. 110, no. 17, pp. 6985-6990, 2013. 


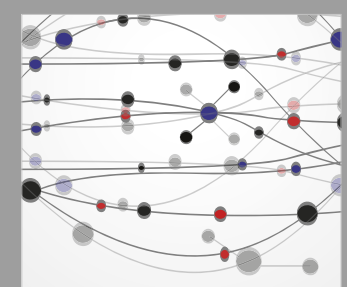

The Scientific World Journal
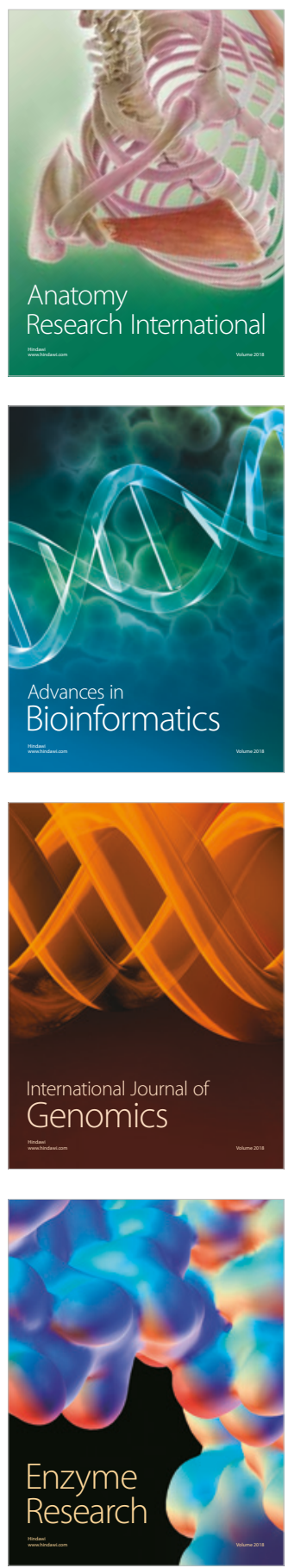
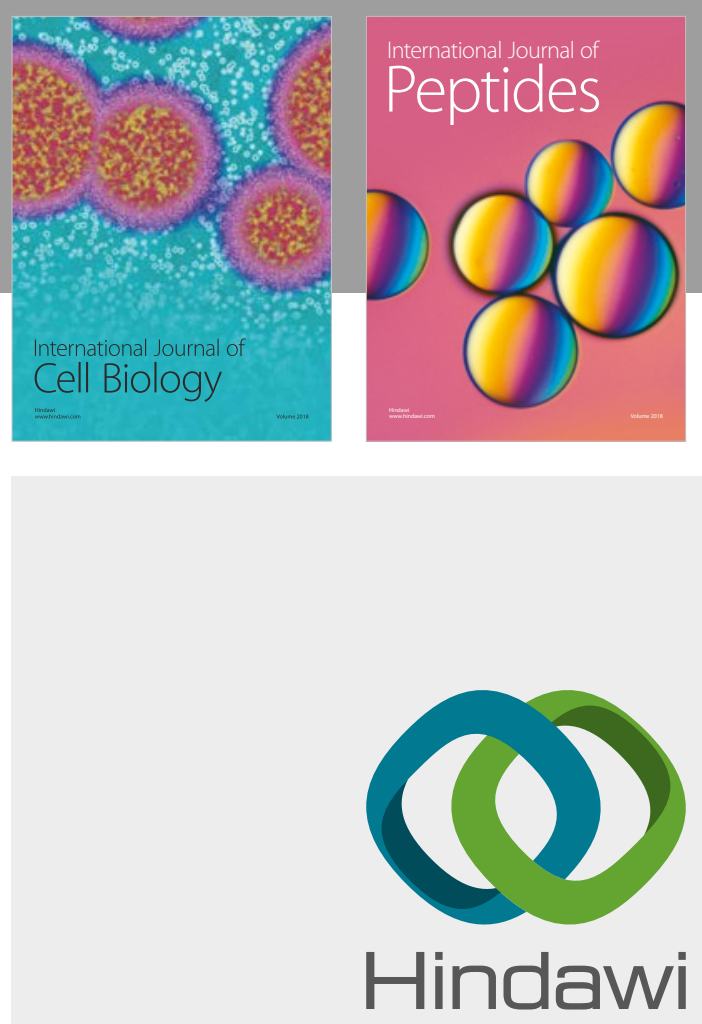

Submit your manuscripts at

www.hindawi.com
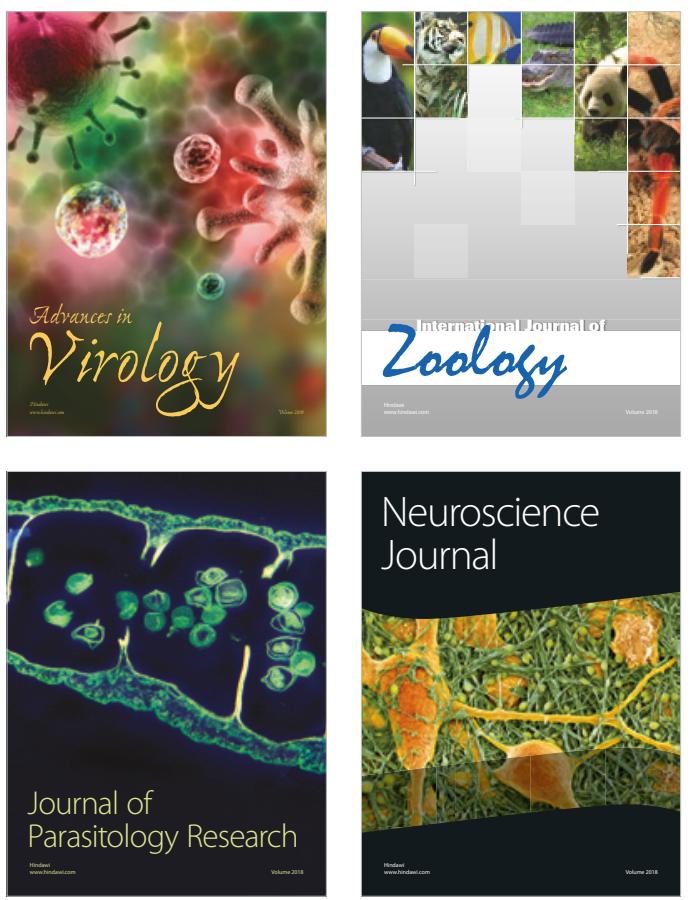
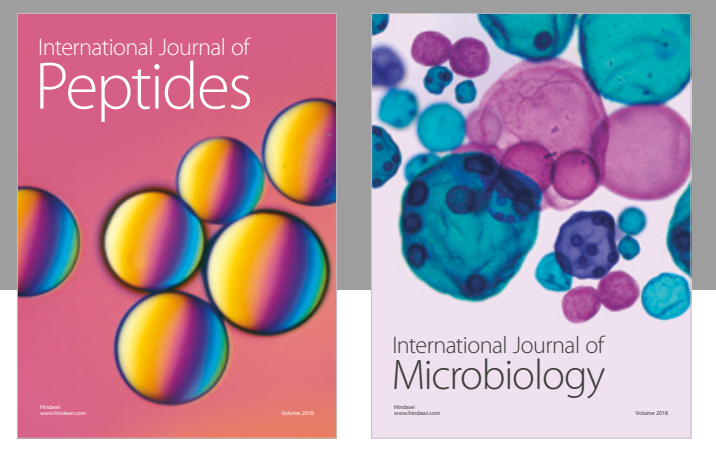

nternational Journal of Microbiology
Journal of
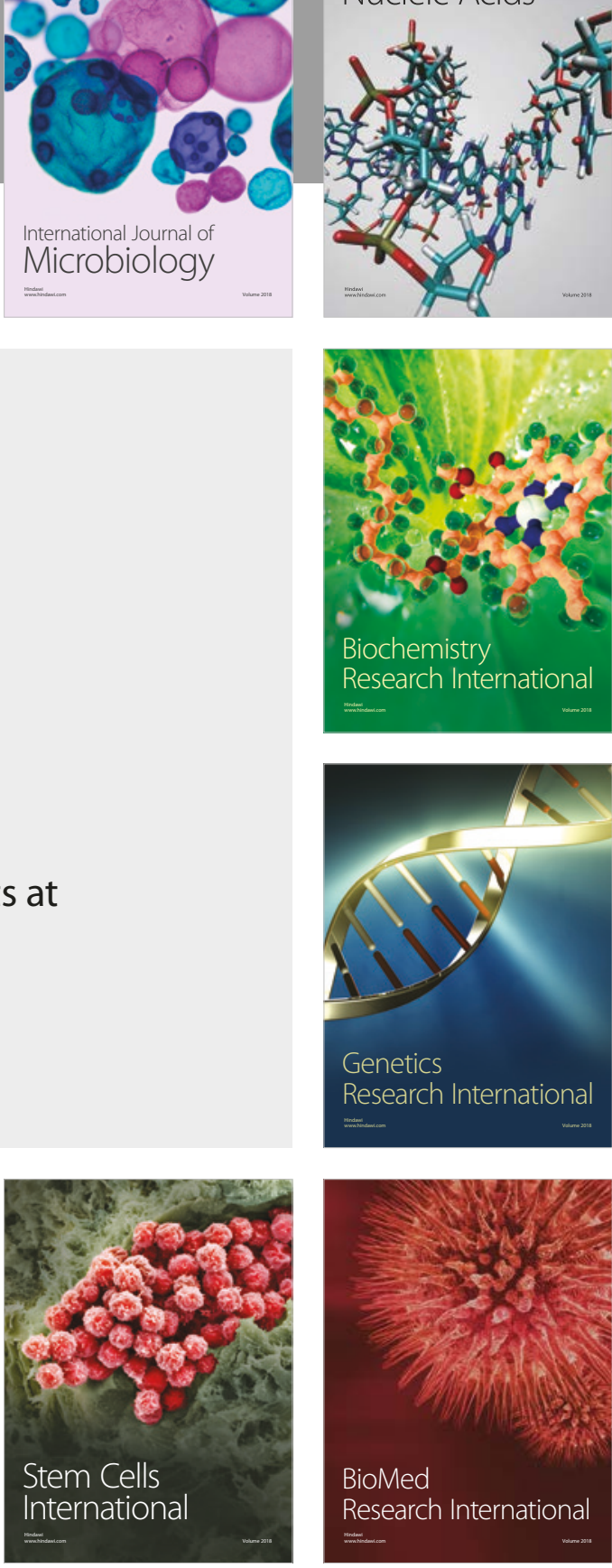
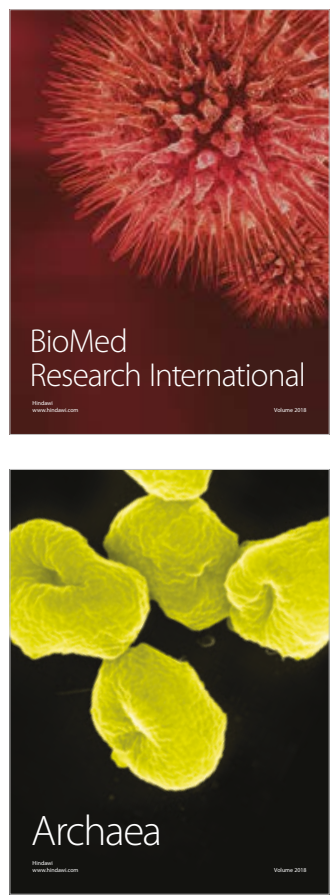